\title{
EMPILEMENTS DE CERCLES ET MODULES COMBINATOIRES
}

\author{
PETER HAÏSSINSKY
}

Résumé.

Le but cette note est de tenter d'expliquer les liens étroits qui unissent la théorie des empilements de cercles et des modules combinatoires, et de comparer les approches à la conjecture de J.W. Cannon qui en découlent $[11,9,10,4,5,6]$.

\section{Abstract.}

The aim of this article is to explain the deep relationships between circle-packings and combinatorial moduli of curves, and to compare the approaches to Cannon's conjecture to which they lead $[11,9,10,4,5,6]$.

\section{TABLE DES MATIÈRES}

INTRODUCTION 3

1. Empilements de cercles 4

2. Modules combinatoires 5

2.1. Modules dans un graphe 5

2.2. Modules sur une surface 11

2.3. Comparaisons des modules 13

3. Théorème de Riemann combinatoire et empilements de cercles 16

3.1. Théorème de Riemann combinatoire revisité 16

3.2. Paramétrage quasisymétrique de sphères topologiques 22

3.3. Remarque sur l'utilisation des toitures 29

3.4. Sur les démonstrations des différentes versions du théorème de Riemann 30

4. Applications à la conjecture de Cannon 31

Date: Version du 20 septembre 2018.

2000 Mathematics Subject Classification. 52C26 30C62 (30F10 30F40).

Key words and phrases. empilement de cercles/circle packings, quasiconforme/quasiconformal, module de courbes/modulus of curves. 
4.1. Approche de Cannon et al. 32

4.2. Approche de Bonk et Kleiner 32

4.3. En guise de conclusion 33

Annexe A. Notions de théorie géométrique des fonctions 34

Annexe B. Discrétisation dans les espaces métriques 39

Annexe C. Convergence et topologie de Hausdorff 45

Références $\quad 49$ 


\section{INTRODUCTION}

Plusieurs approches ont été développées pour estimer des modules de courbes dans des espaces topologiques munis de structures très faibles. Les premiers résultats dans ce sens sont certainement dûs à P. Pansu qui généralise la notion de module dans le cadre d'espaces munis de familles (indénombrables) de 《boules» [20]. D'autres méthodes ont aussi été développées par J. Heinonen et P. Koskela notamment pour étudier les homéomorphismes quasiconformes et leurs avatars dans des espaces de Carnot, voire dans des espaces métriques plus généraux [15].

L'objet de ce texte est d'unifier autant que possible les approches de J.W. Cannon, W. Floyd et W. Parry et de M. Bonk et B. Kleiner à des problèmes d'uniformisation de surfaces motivés par la conjecture de Cannon qui affirme qu'un groupe hyperbolique dont le bord est homéomorphe à $\mathbb{S}^{2}$ admet une action kleinéenne cocompacte. Ces deux approches reposent sur des notions de modules combinatoires légèrement différentes qui conduisent à des résultats apparemment similaires. Le résultat central de cet article est le Théorème 3.4 qui fournit un socle commun pour en déduire d'une part une version faible $\mathrm{du}$ théorème de Riemann combinatoire de J.W. Cannon (Corollaire 3.10) et d'autre part les résultats de M. Bonk et B. Kleiner (voir p.ex. le Corollaire 3.13). Le point clef des démonstrations que l'on présente est d'utiliser, à l'instar de M. Bonk et B. Kleiner, les empilements de cercles pour traduire naturellement les données combinatoires en données analytiques.

Pour ce faire, plusieurs variantes de modules combinatoires présentés par ces différents auteurs sont développées dans ce texte, et on établit quelques comparaisons entre elles. Nous adoptons le point de vue du lecteur familier des notions et techniques de théorie géométrique des fonctions pour démontrer les propriétés de ces modules. Ces notions sont rappelées brièvement dans l'Appendice $\mathrm{A}$, où quelques démonstrations sont aussi esquissées pour insister sur le traitement commun de ces deux théories.

Ce texte est organisé en quatre parties et trois appendices. Les deux premières parties fournissent des rappels concernant les empilements de cercles d'une part, et les modules combinatoires de courbes d'autre part. La troisième partie contient les résultats proprement dits de l'article et la comparaison des deux méthodes de discrétisation. La dernière partie contient une discussion sommaire sur les approches à la conjecture de Cannon. L'Appendice A décrit succinctement les notions de théorie géométrique des fonctions qui sont utilisées tout au long du texte, et qui servent de motivation. Dans l'Appendice B, on montre 
que ces méthodes de discrétisation sont aussi intéressantes dans le cadre d'espaces métriques plus généraux. Enfin, dans l'Appendice C, on établit un critère de convergence pour des fonctions définies sur des sous-ensembles compacts d'un espace compact métrique qui converge au sens de Hausdorff.

Les résultats de ce texte sont essentiellement connus, mais il est espéré qu'ils sont mis en perspective de manière profitable, et que les arguments présentés seront trouvés plus simples.

Remerciements. Je remercie J. Los, L. Reeves et H. Short pour les nombreuses discussions que nous avons eues autour des thèmes abordés dans ce texte, sans lesquelles ce travail n'aurait pu voir le jour. Je remercie aussi Hadrien Larôme qui a relu avec beaucoup d'attention une grande partie de ce manuscrit. Je suis reconnaissant au rapporteur pour ses nombreux conseils qui m'ont permis d'améliorer cet article.

Notations. L'ensemble des réels positifs ou nul est désigné par $\mathbb{R}_{+}$. Dans tout le texte, si $a$ et $b$ sont strictement positifs, on écrit $a \asymp b$ s'il existe une constante universelle $u>1$ telle que $(1 / u) \cdot a \leq b \leq u \cdot a$, $a \lesssim b$ ou $b \gtrsim a$ s'il existe une constante universelle $C>1$ telle que $a \leq C \cdot b$.

\section{EMPILEMENTS DE CERCLES}

Un empilement de cercles $\mathcal{E}$ de la sphère de Riemann $\widehat{\mathbb{C}}$ est une collection de disques fermés d'intérieurs deux à deux disjoints telle que les composantes connexes du complémentaire de la réunion de ces disques soient bordées par exactement trois arcs de cercle.

A un empilement $\mathcal{E}$, on associe son graphe d'incidence $G(\mathcal{E})$ dont les sommets sont les disques et les arêtes sont les paires de disques dont les fermetures s'intersectent. Lorsque $\mathcal{E}$ est fini, il s'agit d'une triangulation que l'on peut voir plongée dans la surface.

Nous énonçons dès maintenant un théorème de représentation conforme pour les empilements qui correspond à une réciproque du fait précédent.

Théorème 1.1 (P. Koebe [18]). Soit $\mathcal{T}$ une triangulation de la sphère $\mathbb{S}^{2}$. Il existe un unique empilement de cercles $\mathcal{E}$ de $\widehat{\mathbb{C}}$ de graphe d'incidence isomorphe à $\mathcal{T}$, à transformation homographique près.

Une démonstration plus moderne figure dans [12].

W.P. Thurston a proposé de démontrer le théorème de représentation conforme de Riemann en utilisant les empilements de cercles. Soient $\Omega \subset \mathbb{C}$ un domaine simplement connexe borné du plan, et $z_{1}, z_{2} \in \Omega$ deux points distincts. Pour $r>0$, on note $\mathcal{T}_{r}$ la triangulation de $\mathbb{C}$ 
par triangles équilatéraux engendrée par $[0, r] \times\{0\}$. On considère la réunion des triangles pleins entièrement contenus dans $\Omega$, et on désigne par $\Omega_{r}$ la composante connexe de son intérieur qui contient $z_{1}$. Si $r$ est assez petit, alors $z_{2}$ en fait aussi partie. Soit $\mathcal{T}_{r}(\Omega)=\mathcal{T}_{r} \cap \bar{\Omega}_{r}$ la restriction de la triangulation $\mathcal{T}_{r}$ à $\overline{\Omega_{r}}$. On ajoute à $\mathcal{T}_{r}(\Omega)$ un sommet (abstrait) et des arêtes qui le relient aux sommets du bord de $\mathcal{T}_{r}(\Omega)$. On obtient ainsi une triangulation de la sphère, pour laquelle on peut appliquer le Théorème 1.1 afin d'obtenir un empilement de cercles $\mathcal{E}_{r}$ dont le complémentaire du disque unité $\mathbb{C} \backslash \mathbb{D}$ est associé au sommet rajouté. On note $f_{r}: \mathcal{T}_{r}(\Omega) \rightarrow \mathbb{D}$ l'application simpliciale induite par les triangulations sous-jacentes. Quitte à composer par un automorphisme de $\mathbb{D}$, on peut supposer que $f_{r}\left(z_{1}\right)=0$ et $f_{r}\left(z_{2}\right)>0$. Le but est alors de montrer que $f_{r}$ converge vers l'application de Riemann quand $r$ tend vers 0 , ce qu'ont fait B. Rodin et D. Sullivan [21].

Théorème 1.2. Le chemin $\left(f_{r}\right)_{r}$ converge uniformément sur les compacts de $\Omega$ vers la transformation conforme $f: \Omega \rightarrow \mathbb{D}$ telle que $f\left(z_{1}\right)=0$ et $f\left(z_{2}\right)>0$ lorsque $r$ tend vers 0 .

Un point crucial de leur démonstration est de montrer que la suite est uniformément quasiconforme (voir Appendice A). Cette propriété est une conséquence du lemme suivant.

Lemme 1.3 (du collier). Il existe une constante $C=C(N)>0$ telle que si un disque $D$ de rayon $r$ d'un empilement de cercles a $N$ voisins, alors le rayon $r^{\prime}$ de chaque voisin vérifie $r^{\prime}>C(N) r$.

Une démonstration figure dans [21].

\section{Modules COMBinatoires}

La notion classique de modules de courbes est exposée dans l'Appendice A. On s'en inspire pour en développer une théorie combinatoire.

2.1. Modules dans un graphe. Soit $G$ un graphe connexe de sommets $\mathcal{S}$ et d'arêtes $\mathcal{A}$. On munit ce graphe d'une métrique qui rend chaque arête isométrique au segment $[0,1]$. Une courbe sur le graphe sera un sous-graphe connexe de $G$, donc ses sommets sont dans $\mathcal{S}$, et ses arêtes dans $\mathcal{A}$.

Pour $Q \geq 1$, on note $\mathcal{M}_{Q}(G)$ l'ensemble des applications $\rho: \mathcal{S} \rightarrow$ $\mathbb{R}_{+}$telles que $0<\sum \rho(s)^{Q}<\infty$ que l'on appelle poids ou métriques admissibles, ou $Q$-admissibles pour être plus précis.

Soit $K$ un ensemble de sommets de $G$; la $\rho$-longueur de $K$ est par définition

$$
\ell_{\rho}(K)=\sum_{s \in K} \rho(s)
$$


et son $Q$-volume

$$
V_{Q, \rho}(K)=\sum_{s \in K} \rho(s)^{Q}
$$

Lorsque $K=\mathcal{S}$, on notera plus simplement $V_{Q, \rho}(\mathcal{S})=V_{Q}(\rho)$.

Si $\Gamma$ est une famille de courbes dans $G$ et $\rho$ est une métrique $Q$ admissible, on définit

$$
L_{\rho}(\Gamma, \mathcal{S})=\inf _{\gamma \in \Gamma} \ell_{\rho}(\gamma)
$$

et son $Q$-module combinatoire par

$$
\bmod _{Q}(\Gamma, G)=\inf _{\rho \in \mathcal{M}_{Q}(G)} \frac{V_{Q}(\rho)}{L_{\rho}(\Gamma, \mathcal{S})^{Q}}=\inf _{\rho \in \mathcal{M}_{Q}(G)} \bmod _{Q}(\Gamma, \rho, \mathcal{S}) .
$$

Si, pour une métrique $\rho$, on a $L_{\rho}(\Gamma, \mathcal{S})=0$, alors on pose $\bmod _{Q}(\Gamma, \rho, \mathcal{S})=$ $+\infty$.

On peut remarquer que si $\lambda>0$, alors $\bmod _{Q}(\Gamma, \lambda \rho, \mathcal{S})=\bmod _{Q}(\Gamma, \rho, \mathcal{S})$, ce qui permet de normaliser les métriques par des conditions du type $L_{\rho}(\Gamma, \mathcal{S}) \geq 1$, ou encore $V_{Q, \rho}(G)=1$.

On a les propriétés suivantes :

(1) si $\Gamma_{1} \subset \Gamma_{2}, \bmod _{Q}\left(\Gamma_{1}, G\right) \leq \bmod _{Q}\left(\Gamma_{2}, G\right)$;

(2) $\bmod _{Q}\left(\bigcup_{i=1}^{\infty}\left(\Gamma_{i}, G\right) \leq \sum_{i=1}^{\infty} \bmod _{Q}\left(\Gamma_{i}, G\right)\right.$;

(3) si $\Gamma_{1}$ et $\Gamma_{2}$ sont deux familles de courbes telles que toute courbe $\gamma_{1}$ dans $\Gamma_{1}$ possède une sous-courbe $\gamma_{2} \in \Gamma_{2}$, alors $\bmod _{Q}\left(\Gamma_{1}, G\right) \leq$ $\bmod _{Q}\left(\Gamma_{2}, G\right)$.

En supposant $\mathcal{S}$ fini et en normalisant les éléments de $\mathcal{M}_{Q}(G)$ pour que $\sum \rho(s)^{Q}=1$, il n'est pas difficile de montrer l'existence d'une métrique extrémale i.e., pour laquelle l'infimum est atteint. Voici une adaptation au cas discret d'un critère de A. Beurling d'extrémalité (voir l'Appendice A).

Proposition 2.1 (critère de Beurling). Soient $G$ un graphe fini et connexe, $\Gamma$ une famille de courbes et $Q>1$. Une métrique $\rho$ est extrémale si et seulement si il existe une sous-famille $\Gamma_{0} \subset \Gamma$ telle que

(1) pour toute $\gamma \in \Gamma_{0}, \ell_{\rho}(\gamma)=L_{\rho}(\Gamma, \mathcal{S})$;

(2) pour toute $h: \mathcal{S} \rightarrow \mathbb{R}$ telle que, pour $\gamma \in \Gamma_{0}, \sum_{s \in \mathcal{S}(\gamma)} h(s) \geq 0$, on ait $\sum_{s \in \mathcal{S}} h(s) \rho(s)^{Q-1} \geq 0$, où $\mathcal{S}(\gamma)$ désigne les sommets $s \in$ $\mathcal{S}$ qui intersectent $\gamma$.

De plus, cette métrique est unique à normalisation près. 
Il est pratique d'introduire à ce niveau quelques notations et de noter quelques observations. Si $G=(\mathcal{S}, \mathcal{A})$ est un graphe fini et $\Gamma$ une famille de courbes, on notera $\Gamma(s)$ l'ensemble des courbes de $\Gamma$ qui contiennent l'élément $s \in \mathcal{S}$; de même, $\mathcal{S}(\gamma)$ désignera l'ensemble des sommets de $\mathcal{S}$ contenus dans la courbe $\gamma \in \Gamma$. Remarquons que si $\rho$ est extrémale, alors pour tout sommet $s$ du support supp $\rho$ de $\rho$, il existe une courbe $\gamma \in \Gamma$ telle que $\ell_{\rho}(\gamma)=L_{\rho}(\Gamma, \mathcal{S})$, car sinon on pourrait diminuer la valeur de $\rho(s)$, ce qui diminuerait $\bmod _{Q}(\Gamma, \rho, \mathcal{S})$.

Enfin, on considère l'espace vectoriel réel $\mathbb{R}^{\mathcal{S}}$ muni de sa base canonique $\left\{e_{s}\right\}_{s \in \mathcal{S}}$ et du produit scalaire $\langle\cdot, \cdot\rangle$ qui rend cette base orthonormée. A chaque courbe $\gamma \in \Gamma$, on associe le vecteur $u_{\gamma}=\sum_{s \in \mathcal{S}(\gamma)} e_{s}$. Une métrique $\sigma$ est donc un vecteur de $\mathbb{R}^{\mathcal{S}}$ et si $\gamma \in \Gamma$, alors $\ell_{\sigma}(\gamma)=$ $\left\langle u_{\gamma}, \sigma\right\rangle$. On définit aussi $v(\sigma)=\sum_{s \in \mathcal{S}}|\sigma(s)|^{Q}$ et on note que $v: \mathbb{R}^{\mathcal{S}} \rightarrow \mathbb{R}$ est strictement convexe si $Q>1$ et linéaire si $Q=1$. En tant que fonction numérique définie sur l'espace vectoriel $\mathbb{R}^{\mathcal{S}}, v$ est différentiable et son gradient $\nabla_{\rho} v$ en un vecteur $\rho$ a pour coordonnées $Q \rho(s)|\rho(s)|^{Q-2}$, $s \in \mathcal{S}$.

Lemme 2.2. Avec les notations ci-dessus et $Q>1$, si $\rho$ est extrémale, alors il existe une sous-famille $\Gamma_{0} \subset \Gamma$ et un vecteur $\left(\lambda_{\gamma}\right) \in\left(\mathbb{R}_{+}\right)^{\Gamma_{0}}$ tels que

(1) pour toute $\gamma \in \Gamma_{0}, \ell_{\rho}(\gamma)=L_{\rho}(\Gamma, \mathcal{S})$;

(2) $\nabla_{\rho} v=\sum_{\gamma \in \Gamma_{0}} \lambda_{\gamma} u_{\gamma}$.

Ce lemme apparaît dans [9] dans le cas $Q=2$.

DÉmonstration. Tout d'abord, on note $\Gamma_{0}$ l'ensemble des courbes de $\Gamma$ de longueur minimale, que l'on fixe égale à 1 . Notons $\delta=\inf _{\Gamma \backslash \Gamma_{0}} \ell_{\rho}(\gamma)-$ 1. Puisque l'on a un nombre fini de vecteurs $u_{\gamma}$ possibles, on a $\delta>0$.

On considère l'ensemble $\Delta$ des vecteurs $\sigma \in \mathbb{R}^{\mathcal{S}}$ tels que $\ell_{\sigma}(\gamma)=1$ pour toutes les courbes $\gamma \in \Gamma_{0}$. Il s'agit d'un espace affine, donc la restriction de $v$ à $\Delta$ admet un unique minimum en un point $\rho_{1}$ car $v$ est strictement convexe. Montrons que $\rho_{1}=\rho$ par l'absurde. Pour $t \in[0,1]$, on note $\rho_{t}=\rho+t\left(\rho_{1}-\rho\right)$. Donc, si $\gamma \in \Gamma_{0}$ alors $\ell_{\rho_{t}}(\gamma)=1$, et sinon

$$
\ell_{\rho_{t}}(\gamma) \geq(1-t)(1+\delta)+t \ell_{\rho_{1}}(\gamma)
$$

Du coup, si $t$ est assez petit, alors on a $L_{\rho_{t}}(\Gamma, \mathcal{S}) \geq 1$, et par convexité, on devrait avoir $v\left(\rho_{t}\right)<v(\rho)$, ce qui contredit l'extrémalité de $\rho$. Par suite, $\rho$ est la solution du problème variationnel qui consiste à minimiser $v \operatorname{sur} \Delta$.

La théorie des extrema liés indique que $\nabla_{\rho} v$ est orthogonal à $\Delta$, autrement dit, il existe des multiplicateurs de Lagrange $\left(\lambda_{\gamma}\right) \in \mathbb{R}^{\Gamma_{0}}$ tels 
que

$$
\nabla_{\rho} v=\sum_{\gamma \in \Gamma_{0}} \lambda_{\gamma} u_{\gamma}
$$

Pour conclure, il reste à montrer que l'on peut choisir ces multiplicateurs positifs ou nuls. On procède par l'absurde en montrant que $\rho$ n'est pas extrémale s'il existe un multiplicateur strictement négatif. On note $E$ le sous-espace de $\mathbb{R}^{\mathcal{S}}$ engendré par $\left\{u_{\gamma}, \gamma \in \Gamma_{0}\right\}$ et $F$ l'orthogonal de $\nabla_{\rho} v$ dans $E$ et on définit l'espace affine $A=\nabla_{\rho} v+F$.

L'espace $A$ s'identifie avec un sous-espace de l'espace projectif $\mathbb{P}(E)$ de $E$. Puisque le produit scalaire des $u_{\gamma}$ par $\nabla_{\rho} v$ est toujours positif, chaque demi-droite de vecteur directeur $u_{\gamma}$ coupe $F$ en un point $p_{\gamma}$, et on note 0 le point qui correspond à $\nabla_{\rho} v$.

Si $\nabla_{\rho} v$ n'est pas dans le cône engendré par $\left\{u_{\gamma}\right\}_{\gamma \in \Gamma_{0}}$, alors 0 n'est pas dans l'enveloppe convexe des points $p_{\gamma}$. Du coup, ces points sont dans un demi-espace de $A$ et il existe un vecteur $\sigma \in F$ dont le produit scalaire avec tous les $u_{\gamma}$ est strictement positif, et même minoré par une constante $\eta>0$, puisqu'ils sont en nombre fini.

Posons $\rho_{t}=\rho+t \sigma$ avec $t>0$. Pour $\gamma \in \Gamma_{0}$, on a

$$
\ell_{\rho_{t}}(\gamma)=\ell_{\rho}(\gamma)+t\left\langle u_{\gamma}, \sigma\right\rangle \geq 1+t \eta \text {. }
$$

Et si $\gamma \in \Gamma \backslash \Gamma_{0}, \ell_{\rho_{t}}(\gamma) \geq 1+\delta+t\left\langle u_{\gamma}, \sigma\right\rangle \geq 1+t \eta$ si $t$ est assez petit, donc $L_{\rho_{t}}(\Gamma) \geq 1+\eta t$ si $t$ est assez petit.

Si $t$ assez petit, alors pour tout $s \in \mathcal{S}$ dans le support de $\rho$, on a $\rho_{t}(s)>0$. Si $\rho(s)=0$, alors $\left|\rho_{t}(s)\right|^{Q}=o(t)$. Par conséquent

$$
\begin{aligned}
v\left(\rho_{t}\right) & =\sum_{s \in \mathcal{S}}|\rho(s)+t \sigma(s)|^{Q} \\
& =\sum_{s \in \operatorname{supp} \rho} \rho(s)^{Q}\left(1+Q t \sigma(s) \rho(s)^{-1}+o(t)\right)+o(t) \\
& =v(\rho)+\sum_{s \in \mathcal{S}} Q \rho(s)^{Q-1} t \sigma(s)+o(t) \\
& =v(\rho)+t\left\langle\nabla_{\rho} v, \sigma\right\rangle+o(t) \\
& =v(\rho)+o(t),
\end{aligned}
$$

car $\nabla_{\rho} v$ et $\sigma$ sont orthogonaux par définition.

Donc

$$
\bmod _{Q}\left(\Gamma, \rho_{t}, \mathcal{S}\right) \leq \frac{v(\rho)+o(t)}{(1+\eta t)^{Q}}=\bmod _{Q}(\Gamma, \rho, \mathcal{S})(1-Q \eta t+o(t))+o(t)
$$


ce qui contredit l'extrémalité de $\rho$. Donc $\nabla_{\rho} v$ est dans le cône engendré par $\left\{u_{\gamma}\right\}_{\gamma \in \Gamma_{0}}$ et il existe $\left(\lambda_{\gamma}\right) \in\left(\mathbb{R}_{+}\right)^{\Gamma}$ tel que $\nabla_{\rho} v=\sum_{\gamma \in \Gamma_{0}} \lambda_{\gamma} u_{\gamma}$.

DÉmonstration de la Proposition 2.1. Supposons que $\rho$ vérifie les conditions de A. Beurling. Soit $\sigma \in \mathcal{M}_{Q}(G)$ que l'on suppose normalisée pour que $L_{\sigma}(\Gamma)=L_{\rho}(\Gamma)$. Du coup, $\ell_{\sigma}(\gamma) \geq L_{\rho}(\Gamma)=\ell_{\rho}(\gamma)$ pour toute $\gamma \in \Gamma_{0}$. Donc, en posant $h=\sigma-\rho$, on obtient

$$
\sum_{s \in \mathcal{S}}(\sigma-\rho) \rho^{Q-1} \geq 0
$$

soit

$$
\sum_{s \in \mathcal{S}} \rho^{Q} \leq \sum_{s \in \mathcal{S}} \sigma \rho^{Q-1} \leq\left(\sum_{s \in \mathcal{S}} \sigma^{Q}\right)^{1 / Q} \cdot\left(\sum_{s \in \mathcal{S}} \rho^{Q}\right)^{1-1 / Q}
$$

par l'inégalité de Hölder. Du coup, on $\bmod _{Q}(\Gamma, \rho, \mathcal{S}) \leq \bmod _{Q}(\Gamma, \sigma, \mathcal{S})$. Le cas d'égalité se produit lorsque l'inégalité de Hölder est une égalité, donc si $\sigma^{Q}$ et $\left(\rho^{Q-1}\right)^{Q /(Q-1)}$ sont proportionnelles, soit si $\sigma=\rho$ d'après leurs normalisations.

Réciproquement, supposons que $\rho$ est extrémale et normalisons-la pour que $L_{\rho}(\Gamma)=1$. D'après le lemme précédent, il existe $\Gamma_{0} \subset \Gamma$ et $\left(\lambda_{\gamma}\right) \in\left(\mathbb{R}_{+}\right)^{\Gamma}$ tels que

(1) pour toute $\gamma \in \Gamma_{0}, \ell_{\rho}(\gamma)=L_{\rho}(\Gamma, \mathcal{S})$;

(2) $\nabla_{\rho} v=\sum_{\gamma \in \Gamma_{0}} \lambda_{\gamma} u_{\gamma}$.

Si $h: \mathcal{S} \rightarrow \mathbb{R}$ vérifie $\sum_{s \in \mathcal{S}(\gamma)} h(s) \geq 0$ pour toute courbe $\gamma \in \Gamma_{0}$, alors

$$
\begin{aligned}
\sum_{s \in \mathcal{S}} h(s) \rho(s)^{Q-1} & =\sum_{s \in \mathcal{S}} h(s)\left(\frac{1}{Q} \sum_{\gamma \in \Gamma_{0}(s)} \lambda_{\gamma}\right) \\
& =\frac{1}{Q} \sum_{\gamma \in \Gamma_{0}} \lambda_{\gamma}\left(\sum_{s \in \mathcal{S}(\gamma)} h(s)\right) \\
& \geq 0 .
\end{aligned}
$$

Ceci établit la réciproque.

Condensateurs dans un graphe. Si $E$ et $F$ sont deux ensembles de sommets disjoints d'un graphe $G$, on considère la famille $\Gamma(E, F, G)=$ $\Gamma(E, F)$ des courbes qui joignent $E$ à $F$ et on définit le module, aussi appelé capacité, par

$$
\bmod _{Q}(E, F, G)=\bmod _{Q}(\Gamma(E, F), G) .
$$


On dira que $(E, F)$ est un condensateur si $E$ et $F$ sont les sommets de sous-graphes connexes de $G$.

La capacité dépend de manière essentielle des arêtes du graphe. Cependant, on a le résultat suivant.

Proposition 2.3. Soient $\mathcal{S}$ un ensemble de sommets et $\mathcal{A}_{1}$ et $\mathcal{A}_{2}$ deux familles d'arêtes telles que les graphes associés $G_{1}=\left(\mathcal{S}, \mathcal{A}_{1}\right)$ et $G_{2}=$ $\left(\mathcal{S}, \mathcal{A}_{2}\right)$ soient connexes. Pour $j=1,2$, on munit $G_{j}$ de la distance de longueur $|\cdot|_{j}$ pour laquelle chaque arête est de longueur 1 . On suppose que $\mathcal{A}_{2} \subset \mathcal{A}_{1}, G_{2}$ est de valence bornée par $K$, et que si $\left|v_{1}-v_{2}\right|_{1}=1$ alors $\left|v_{1}-v_{2}\right|_{2} \leq k$. Soit $Q \geq 1$.

(1) Pour toute paire de sommets disjoints $(E, F)$, on a

$$
\bmod _{Q}\left(E, F, G_{1}\right) \asymp \bmod _{Q}\left(E, F, G_{2}\right),
$$

où les constantes implicites ne dépendent que de $k, K$ et $Q$.

(2) $S i\left(E_{1}, F_{1}\right)$ est un condensateur de $G_{1}$, tel que $E_{1}$ et $F_{1}$ sont $\grave{a}$ distance au moins $2 k+1$ dans $G_{1}$, alors il existe un condensateur $\left(E_{2}, F_{2}\right)$ de $G_{2}$, tel que $E_{1} \subset E_{2}, F_{1} \subset F_{2}$ et

$$
\bmod _{Q}\left(E_{1}, F_{1}, G_{1}\right) \asymp \bmod _{Q}\left(E_{2}, F_{2}, G_{2}\right),
$$

où les constantes implicites ne dépendent que de $k, K$ et $Q$.

DÉmonstration. (1) On note $\Gamma_{1}=\Gamma\left(E, F, G_{1}\right)$ la famille de courbes associée à $\{E, F\}$ dans $G_{1}$ et $\Gamma_{2}=\Gamma\left(E, F, G_{2}\right)$ dans $G_{2}$.

Tout d'abord, on a $\bmod _{Q}\left(\Gamma_{2}, G_{2}\right) \leq \bmod _{Q}\left(\Gamma_{1}, G_{1}\right)$ car il y a plus de chemins dans $G_{1}$.

Réciproquement, on se donne une métrique $\rho_{2}: \mathcal{S} \rightarrow \mathbb{R}_{+}$telle que $L_{\rho_{2}}\left(\Gamma_{2}, G_{2}\right)=1$. On définit $\rho_{1}: \mathcal{S} \rightarrow \mathbb{R}_{+} \operatorname{par} \rho_{1}(s)=\max \left\{\rho_{2}\left(s^{\prime}\right), s^{\prime} \in\right.$ $\left.B_{2}(s, k)\right\}$, où $B_{2}(s, k)$ désigne la boule centrée en $s$ de rayon $k$ pour la distance $|\cdot|_{2}$. Notons $N$ le cardinal maximal d'une boule combinatoire $B_{2}(s, k)$. Si $\gamma_{1}$ est une courbe de $\Gamma_{1}$, on lui associe une courbe $\gamma_{2}$ de $\Gamma_{2}$ passant par au moins les mêmes sommets, et dont les détours sont de longueur au plus $N$. Du coup, pour chaque sommet $s$ de $\gamma_{1}$, on a $\rho_{1}(s) \geq(1 / N) \ell_{\rho_{2}}\left(\gamma_{2} \cap B_{2}(s, k)\right)$ et on en déduit que $L_{\rho_{1}}\left(\Gamma_{1}, G_{1}\right) \geq 1 / N$.

De même, on a

$$
V_{Q}\left(\rho_{1}\right) \leq \sum_{s \in \mathcal{S}} \sum_{v \in B_{2}(s, k)} \rho_{2}(v)^{Q} \leq N \sum_{s \in \mathcal{S}} \rho_{2}(s)^{Q} \leq N \cdot V_{Q}\left(\rho_{2}\right)
$$

car chaque $v$ ne peut apparaître qu'au plus $N$ fois. On en déduit $\bmod _{Q}\left(\Gamma_{2}, G_{2}\right) \gtrsim \bmod _{Q}\left(\Gamma_{1}, G_{1}\right)$.

(2) Si $E_{1}$ n'est pas connexe dans $G_{2}$, alors il existe deux sommets $s, s^{\prime} \in E_{1}$ tels que $\left|s-s^{\prime}\right|_{1}=1$ mais $\left|s-s^{\prime}\right|_{2}>1$. On construit une courbe dans $G_{2}$ de longueur au plus $k$ qui relie $s$ à $s^{\prime}$ que l'on ajoute à $E_{1}$. 
On continue ainsi jusqu'à obtenir $E_{2} \supset E_{1}$ comme sommets d'un sousgraphe connexe de $G_{2}$ dans le $k$-voisinage de $E_{1}$. On procède de même avec $F_{1}$ pour obtenir $F_{2}$. Par hypothèses, $E_{2}$ et $F_{2}$ sont disjoints donc $\left(E_{2}, F_{2}\right)$ est un condensateur de $G_{2}$. D'après (1), il suffit de montrer que $\bmod _{Q}\left(E_{1}, F_{1}, G_{2}\right) \asymp \bmod _{Q}\left(E_{2}, F_{2}, G_{2}\right)$.

Par construction, chaque courbe de $\Gamma_{1}=\Gamma\left(E_{1}, F_{1}, G_{2}\right)$ contient une courbe de $\Gamma_{2}=\Gamma\left(E_{2}, F_{2}, G_{2}\right)$, donc $\bmod _{Q}\left(E_{1}, F_{1}, G_{2}\right) \leq \bmod _{Q}\left(E_{2}, F_{2}, G_{2}\right)$. Pour la réciproque, on considère une métrique $\rho_{1}: \mathcal{S} \rightarrow \mathbb{R}_{+}$telle que $L_{\rho_{1}}\left(\Gamma_{1}, G_{2}\right)=1$. On définit $\rho_{2}: \mathcal{S} \rightarrow \mathbb{R}_{+} \operatorname{par} \rho_{2}(s)=\max \left\{\rho_{1}\left(s^{\prime}\right), s^{\prime} \in\right.$ $\left.B_{2}(s, k)\right\}$.

Si $\gamma_{2}$ est une courbe de $\Gamma_{2}$ d'extrémités $e_{2} \in E_{2}$ et $f_{2} \in F_{2}$, on construit des courbes $\gamma_{e}$ et $\gamma_{f}$ de longueur combinatoire au plus $k$ qui relient $e_{2}$ à $E_{1}$ et $f_{2}$ à $F_{1}$ respectivement. On concatène alors $\gamma_{2}$ à $\gamma_{e}$ et $\gamma_{f}$ pour obtenir une courbe $\gamma_{1}$ de $\Gamma_{1}$.

On remarque que

$$
\rho_{2}\left(e_{2}\right) \geq(1 / N) \sum_{s \in B_{2}\left(e_{2}, k\right)} \rho_{1}(s) \geq(1 / N) \ell_{\rho_{1}}\left(\gamma_{e}\right)
$$

et $\rho_{2}\left(f_{2}\right) \geq(1 / N) \ell_{\rho_{1}}\left(\gamma_{f}\right)$. Du coup, $\ell_{\rho_{2}}\left(\gamma_{2}\right) \geq(1 / N) \ell_{\rho_{1}}\left(\gamma_{1}\right) \geq 1 / N$. On conclut comme au point (1).

Définition 2.4 (graphes équivalents). Sous les conditions de la Proposition 2.3, on dira que $G_{1}$ contient le graphe équivalent $G_{2}$.

2.2. Modules sur une surface. Soit $\mathcal{S}$ un recouvrement localement fini d'un espace métrique $X$. Ses éléments seront appelés parfois des pièces, et la maille de $\mathcal{S}$ sera le plus grand diamètre de ses pièces. Le nerf $\mathcal{N}(\mathcal{S})$ de $\mathcal{S}$ est le graphe dont les sommets sont les éléments de $\mathcal{S}$ et les arêtes sont constituées des paires de pièces qui s'intersectent. On dit qu'un recouvrement est de valence bornée si son nerf est de valence bornée, et l'indice de recouvrement entre deux recouvrements est le nombre maximal de pièces de l'un des recouvrements qui intersectent une même pièce de l'autre. L'indice d'auto-recouvrement est l'indice de recouvrement d'un recouvrement par rapport à lui-même. En particulier, un graphe de valence bornée a un indice d'auto-recouvrement fini. On dira dans la suite qu'une suite de recouvrements est de valence bornée si la borne ne dépend pas du recouvrement de la suite. Si $K \subset X$, on note $\mathcal{S}(K)$ l'ensemble des $s \in \mathcal{S}$ tels que $s \cap K \neq \emptyset$.

Par pavage, on entendra un recouvrement localement fini par compacts connexes d'intérieurs deux à deux disjoints.

Une courbe dans un espace topologique $X$ est une application continue $\gamma:[0,1] \rightarrow X$. On ne distinguera pas en général l'application $\gamma$ en tant que telle de son image dans $X$. 
Si $\Gamma$ est une famille de courbes dans $X$, on lui associe une famille de courbes $\widehat{\Gamma}$ dans $\mathcal{N}(\mathcal{S})$ comme suit : chaque courbe $\gamma$ traverse un ensemble de pièces adjacentes $\mathcal{S}(\gamma)$; on associe à $\gamma$ cet ensemble $\mathcal{S}(\gamma)$, ainsi que les arêtes qui les connectent dans le graphe : cela définit une courbe $\widehat{\gamma}$ de $\mathcal{N}(\mathcal{S})$ au sens du paragraphe précédent. On note $\widehat{\Gamma}$ l'ensemble des courbes ainsi obtenues, qui correspond à $\{\mathcal{S}(\gamma), \gamma \in \Gamma\}$ aux arêtes près. On définit

$$
\bmod _{Q}(\Gamma, \mathcal{S})=\bmod _{Q}(\widehat{\Gamma}, \mathcal{N}(\mathcal{S})) .
$$

Si $(E, F)$ est un condensateur i.e., $E$ et $F$ sont deux continua (compacts connexes non dégénérés) disjoints, on définit deux modules. Le premier est associé à la famille des courbes $\Gamma(E, F)$ qui joignent $E$ à $F$ dans $X$, et le second au condensateur vu dans $\mathcal{N}(S)$ : on considère $\widehat{E}=\mathcal{S}(E)$ et $\widehat{F}=\mathcal{S}(F)$ comme sous-ensemble de $\mathcal{N}(\mathcal{S})$.

On définit alors

$$
\left\{\begin{array}{l}
\bmod _{Q}(E, F, \mathcal{S})=\bmod _{Q}(\Gamma(E, F), \mathcal{S}) \\
\widehat{\bmod }_{Q}(E, F, \mathcal{S})=\bmod _{Q}(\widehat{E}, \widehat{F}, \mathcal{N}(\mathcal{S}))
\end{array}\right.
$$

En général, l'inégalité suivante est satisfaite :

$$
\bmod _{Q}(E, F, \mathcal{S}) \leq \widehat{\bmod }_{Q}(E, F, \mathcal{S})
$$

Remarque 2.5. Le module le plus maniable est bien sûr $\widehat{\bmod }_{Q}$ puisqu'il ne dépend que du nerf du recouvrement. En revanche, il peut ne pas porter d'information si par exemple on n'a pas de courbes entre les continua! Du coup, la signification de ce module se fera à travers l'autre, qui lui, dépend plus précisément de la topologie de $X$.

On suppose dorénavant que $X$ est une surface topologique.

Rappelons qu'un quadrilatère est la donnée d'un domaine de Jordan avec quatre points distincts sur le bord définissant ses quatre côtés. Voici l'analogue du Théorème 1.1 ( $c f .[9,22]$ ).

Théorème 2.6. Soit $Q$ un quadrilatère recouvert par un pavage $\mathcal{S}$ dont le nerf forme une triangulation et soit $\Gamma$ la famille de courbes qui joint deux côtés opposés donnés. Il existe un rectangle $R$ et un pavage de nerf identique au précédent par carrés qui respecte les sommets. La longueur des arêtes des carrés produit la métrique extrémale pour $\bmod _{2}(\Gamma, \mathcal{S})$.

Remarque 2.7. Si le pavage ne forme pas une triangulation, alors le pavage par carrés peut avoir un nerf sensiblement différent de l'original si plusieurs pièces de $\mathcal{S}$ s'intersectent en un même point. 
Sur une surface, il est pratique de travailler avec des anneaux, c'est-à dire des surfaces homéomorphes à $] 0,1\left[\times \mathbb{S}^{1}\right.$. On leur associe les modules suivants.

Définition 2.8 (modules combinatoires d'un anneau). Si A un anneau d'une surface recouverte par $\mathcal{S}$, on considère $\Gamma_{t}$ l'ensemble des courbes qui joignent les bords de l'anneau, et $\Gamma_{s}$ la famille des courbes de A qui séparent les composantes de bord. On définit alors

$$
\bmod _{\text {sup }}(A, \mathcal{S})=\frac{1}{\bmod _{2}\left(\Gamma_{t}, \mathcal{S}\right)} \quad \text { et } \bmod _{\text {inf }}(A, \mathcal{S})=\bmod _{2}\left(\Gamma_{s}, \mathcal{S}\right) \text {. }
$$

De plus, si on note $E$ et $F$ les composantes du bord de $A$, on définit aussi $\widehat{\bmod }_{\text {sup }}(A, \mathcal{S})=1 / \widehat{\bmod }_{2}(E, F, \mathcal{S})$. Lorsque $\mathcal{S}$ est planaire, on peut considérer les courbes $\widehat{\Gamma}_{s}$ de $\mathcal{N}(\mathcal{S})$ qui séparent $\mathcal{S}(E)$ et $\mathcal{S}(F)$; on pose alors $\widehat{\bmod }_{\text {inf }}(A, \mathcal{S})=\widehat{\bmod }_{2}\left(\widehat{\Gamma}_{s}, \mathcal{S}\right)$.

2.3. Comparaisons des modules. Pour terminer ce paragraphe, nous donnons quelques propriétés des modules combinatoires qui sont dûs essentiellement à J.W. Cannon, W. Floyd et W. Parry (voir [9] par exemple).

Si deux recouvrements sont d'indice de recouvrement fini, alors les modules associés sont comparables :

Lemme 2.9. Si $\mathcal{S}$ et $\mathcal{S}^{\prime}$ sont deux recouvrements de $X$ d'indice de recouvrement fini $K$, alors, pour toute famille de courbes $\Gamma$ et tout $Q \geq 1$, on $a$

$$
\bmod _{Q}(\Gamma, \mathcal{S}) \asymp \bmod _{Q}\left(\Gamma, \mathcal{S}^{\prime}\right)
$$

où les constantes implicites ne dépendent que de $K$ et de $Q$.

DÉmonstration. Si $\rho$ est une métrique admissible pour $\mathcal{S}$, on définit $\sigma: \mathcal{S}^{\prime} \rightarrow \mathbb{R}_{+}$par

$$
\sigma\left(s^{\prime}\right)=\max \left\{\rho(s), s \in \mathcal{S}, s \cap s^{\prime} \neq \emptyset\right\} .
$$

Du coup, si $\gamma$ est une courbe de $\Gamma$, alors

$$
\ell_{\sigma}\left(\gamma, \mathcal{S}^{\prime}\right) \geq(1 / K) \sum_{s^{\prime} \in \mathcal{S}^{\prime}(\gamma)} \sum_{s \in \mathcal{S}\left(s^{\prime}\right)} \rho(s) \geq(1 / K) \sum_{s \in \mathcal{S}(\gamma)} \rho(s)
$$

donc $\ell_{\sigma}\left(\gamma, \mathcal{S}^{\prime}\right) \geq(1 / K) \ell_{\rho}(\gamma, \mathcal{S})$. D'autre part,

$$
V_{Q}\left(\sigma, \mathcal{S}^{\prime}\right) \leq \sum_{s^{\prime} \in \mathcal{S}^{\prime}} \sum_{s \in \mathcal{S}\left(s^{\prime}\right)} \rho(s)^{Q} \leq K \sum_{s \in \mathcal{S}} \rho(s)^{Q}
$$

donc $V_{Q}\left(\sigma, \mathcal{S}^{\prime}\right) \leq K V_{Q}(\rho, \mathcal{S})$ et on en déduit $\bmod _{Q}\left(\Gamma, \mathcal{S}^{\prime}\right) \lesssim \bmod _{Q}(\Gamma, \mathcal{S})$. On établit le lemme en inversant les rôles de $\mathcal{S}$ et $\mathcal{S}^{\prime}$. 
Définition 2.10 (tuiles, toiture). Une tuile est un compact connexe et une toiture est un recouvrement localement fini par tuiles.

Proposition 2.11. Soit $X$ un espace métrique propre connexe, localement connexe par arcs recouvert par une toiture $\mathcal{S}$ de valence bornée. Alors pour tout condensateur $(E, F)$, on a

$$
\widehat{\bmod }_{Q}(E, F, \mathcal{S}) \asymp \bmod _{Q}(E, F, \mathcal{S})
$$

où les constantes implicites ne dépendent que de la valence.

Remarque 2.12. Lorsque $X$ est une surface et $\mathcal{S}$ est planaire, alors la démonstration ci-dessous montre que $\widehat{\bmod }_{\inf }(A, \mathcal{S}) \asymp \bmod _{\inf }(A, \mathcal{S})$.

DÉmonstration. Tout d'abord, notons que sous les conditions de cette proposition, un ouvert connexe de $X$ est aussi connexe par arcs.

On note $\widehat{\Gamma}$ la famille de courbes dans $\mathcal{N}(\mathcal{S})$ qui joignent $\mathcal{S}(E)$ et $\mathcal{S}(F)$. Si $\rho$ est optimale pour $\bmod _{Q}(E, F, \mathcal{S})$, on définit

$$
\widehat{\rho}(s)=\sum_{s^{\prime} \in B_{1}(s, 1)} \rho(s) .
$$

Soit $\widehat{\gamma} \in \widehat{\Gamma}$. Notons d'une part $L$ la réunion des pièces dans $X$ qui n'intersectent pas $\mathcal{S}(\widehat{\gamma})$ et d'autre part $K$ la réunion des pièces qui forment le support de $\widehat{\gamma}$. Alors $L$ est fermé car $\mathcal{S}$ est localement fini, $K$ est un continuum car $\mathcal{S}$ est une toiture, et $K \subset X \backslash L$. Du coup, la composante connexe de $X \backslash L$ qui contient $K$ contient une courbe $\gamma \in \Gamma(E, F)$. Cette courbe se trouve dans le 1 -voisinage de $K$ dans $\mathcal{N}(\mathcal{S})$. Donc $\ell_{\widehat{\rho}}(\widehat{\gamma}) \geq \ell_{\rho}(\gamma)$.

D'autre part, l'inégalité de Hölder implique

$$
\widehat{\rho}(s)^{Q} \leq(K+1)^{Q-1} \sum_{s^{\prime} \in B(s, 1)} \rho\left(s^{\prime}\right)^{Q}
$$

donc

$$
V_{Q}(\widehat{\rho}) \leq(K+1)^{Q-1} \sum_{s} \sum_{s^{\prime} \in B(s, 1)} \rho\left(s^{\prime}\right)^{Q} \leq(K+1)^{Q} V_{Q}(\rho),
$$

puisque chaque pièce $s^{\prime}$ apparaît dans au plus $(K+1)$ boules distinctes de rayon 1.

Remarque 2.13. Si $\mathcal{S}$ est un pavage par domaines de Jordan alors on obtient $\bmod _{\text {sup }}=\widehat{\bmod }_{\text {sup }}$ et $\bmod _{\text {inf }}=\widehat{\bmod }_{\text {inf }}$. En effet, à chaque courbe $\widehat{\gamma}$ dans le graphe va correspondre une courbe de la surface $\gamma$ telle que $\mathcal{S}(\gamma)=\mathcal{S}(\widehat{\gamma})$.

Nous donnons une nouvelle démonstration du lemme suivant ( $c f .[9])$. 
Lemme 2.14. Si A est un anneau d'une surface $X$ recouverte par $\mathcal{S}$, alors

$$
\bmod _{\text {inf }}(A, \mathcal{S}) \leq \bmod _{\text {sup }}(A, \mathcal{S}) .
$$

DÉMONSTRATION. Il suffit de trouver une métrique admissible $\rho$ pour laquelle $\bmod _{2}\left(\Gamma_{s}, \rho, \mathcal{S}\right) \leq 1 / \bmod _{2}\left(\Gamma_{t}, \rho, \mathcal{S}\right)$. Dans ce sens, nous considérons la métrique extrémale qui nous fournit $\bmod _{\text {sup }}(A, \mathcal{S})$ avec $L_{\rho}\left(\Gamma_{t}, \mathcal{S}\right)=$ 1. D'après le Lemme 2.2 , il existe $\Gamma_{0}$ et des coefficients $\left(\lambda_{\gamma}\right) \in \mathbb{R}^{\Gamma_{0}}$ tels que, pour toute $s \in \operatorname{supp} \rho$,

$$
\rho(s)=(1 / 2) \sum_{\gamma \in \Gamma_{0}(s)} \lambda_{\gamma} .
$$

On remarque qu'une courbe $\widehat{\gamma} \in \Gamma_{s}$ doit couper chaque courbe de $\Gamma_{0}$ par le théorème de Jordan, donc la $\rho$-longueur de $\widehat{\gamma}$ doit être au moins $(1 / 2) \sum_{\Gamma_{0}} \lambda_{\gamma}$ donc

$$
L_{\rho}\left(\Gamma_{s}, \mathcal{S}\right) \geq(1 / 2) \sum_{\Gamma_{0}} \lambda_{\gamma}
$$

D'autre part, on a

$$
\begin{aligned}
\sum_{\mathcal{S}} \rho(s)^{2} & =(1 / 2) \sum_{\mathcal{S}} \rho(s) \sum_{\gamma \in \Gamma_{0}(s)} \lambda_{\gamma} \\
& =(1 / 2) \sum_{\gamma \in \Gamma_{0}} \lambda_{\gamma} \sum_{\mathcal{S}(\gamma)} \rho(s) \\
& =(1 / 2) \sum_{\gamma \in \Gamma_{0}} \lambda_{\gamma} \\
& \leq L_{\rho}\left(\Gamma_{s}\right) .
\end{aligned}
$$

Par conséquent, on obtient

$$
\frac{V_{2}(\rho, \mathcal{S})}{L_{\rho}\left(\Gamma_{s}, \mathcal{S}\right)^{2}} \leq \frac{1}{V_{2}(\rho, \mathcal{S})}
$$

soit

$$
\bmod _{\text {inf }}(A, \mathcal{S}) \leq \bmod _{2}\left(\Gamma_{s}, \rho, \mathcal{S}\right) \leq \frac{1}{\bmod _{2}\left(\Gamma_{t}, \rho, \mathcal{S}\right)}=\bmod _{\text {sup }}(A, \mathcal{S})
$$

Remarque 2.15. Dans [10], les auteurs vont jusqu'à montrer que dans le cas d'une toiture de valence bornée, on a $\bmod _{\mathrm{inf}}=\widehat{\bmod }_{\text {sup }}$. Une version plus faible de ce résultat sera établie au Corollaire 3.8. 


\section{ThÉorème De Riemann COMbinatoire ET EMPILEMEnts DE CERCLES}

Notre objectif ici est de mélanger les approches de J.W. Cannon et de M. Bonk et B. Kleiner pour retrouver l'essentiel de leurs résultats. On commence par une version faible du théorème de Riemann combinatoire qui nous servira ensuite à obtenir des paramétrages quasisymétriques de sphères topologiques soumises à des conditions analytiques.

3.1. Théorème de Riemann combinatoire revisité. On montre une version faible du théorème de Riemann combinatoire (Théorème 3.4) en utilisant les empilements de cercles. Notre approche en redonne une démonstration succincte dans un cadre simplifié.

3.1.1. Le théorème de Riemann combinatoire. Rappelons l'enoncé du théorème original de J.W. Cannon. Pour cela on introduit quelques notions supplémentaires.

Définition 3.1 (approximation séparante). C'est une suite de recouvrements localement finis $\left(\mathcal{S}_{n}\right)_{n}$ dont la maille tend vers 0 telle que

(1) pour tout anneau $A$, il existe $m>0$ et $n_{0}$ telle que, pour $n \geq n_{0}$, on ait

$$
\bmod _{\text {sup }}\left(A, \mathcal{S}_{n}\right) \geq m
$$

(2) pour tout $x \in X$, tout voisinage $V$ de $x$ et tout $m>0$, il existe un anneau $A \subset V$ qui sépare $x$ de $X \backslash V$ et $n_{0}$ tels que

$$
\bmod _{\text {sup }}\left(A, \mathcal{S}_{n}\right) \geq m
$$

pour $n \geq n_{0}$.

Définition 3.2 (approximation conforme). C'est une suite de recouvrements $\left(\mathcal{S}_{n}\right)_{n}$ dont la maille tend vers 0 telle que

(1) la suite est une approximation séparante;

(2) il existe $K \geq 1$ telle que, pour tout anneau $A$ bordé par des courbes de Jordan, il existe $m>0$ et $n_{0}$ telles que, pour $n \geq n_{0}$,

$$
\bmod _{\text {sup }}\left(A, \mathcal{S}_{n}\right), \bmod _{\text {inf }}\left(A, \mathcal{S}_{n}\right) \in[m / K, K m] .
$$

Avec ces notations, on a

Théorème 3.3 (de Riemann combinatoire [8]). Si $\left(\mathcal{S}_{n}\right)$ est une approximation conforme de toitures sur une surface topologique, alors celle-ci admet une structure complexe telle que les modules analytiques des anneaux soient comparables à leurs modules combinatoires pour $n$ 
assez grand i.e., il existe $K^{\prime}$ telle que pour tout anneau $A$, il existe $n_{0}=n_{0}(A)$ et $m>0$ telles que si $n \geq n_{0}$ alors

$$
\bmod _{\text {sup }}\left(A, \mathcal{S}_{n}\right), \bmod _{\mathrm{inf}}\left(A, \mathcal{S}_{n}\right), \bmod _{2} A \in\left[m / K^{\prime}, K^{\prime} m\right] .
$$

3.1.2. Une version sphérique. Soit $X$ une surface topologique homéomorphe à $\mathbb{S}^{2}$ que l'on suppose métrisée. Soit $\left(\mathcal{S}_{n}\right)_{n}$ une suite de toitures dont la maille tend vers 0 et telle que le nerf $\mathcal{N}\left(\mathcal{S}_{n}\right)$ de $\mathcal{S}_{n}$ contient une triangulation $\mathcal{T}_{n}$ de $\mathbb{S}^{2}$ équivalente au sens de la définition 2.4 pour chaque $n$. Observons que l'existence d'un recouvrement dont le nerf est une triangulation provient du fait que $X$ est de dimension topologique 2, voir [19].

On remarque que puisque les triangulations sont planaires, on peut aussi considérer $\widehat{\bmod }_{\text {inf }}\left(\cdot, \mathcal{T}_{n}\right)$. Cependant, $\mathcal{N}\left(\mathcal{S}_{n}\right)$ peut ne pas être planaire ; c'est pourquoi on définit $\widehat{\bmod }_{\text {inf }}\left(\cdot, \mathcal{S}_{n}\right)=\widehat{\bmod }_{\text {inf }}\left(\cdot, \mathcal{T}_{n}\right)$ par commodité.

On se fixe trois points distincts $a_{1}, a_{2}, a_{3} \in X$. Pour chaque $n \geq 0$ et chaque $s \in \mathcal{S}_{n}$, on se donne $p(s) \in s$ et on note $\mathcal{P}_{n}$ l'ensemble des ces points. On les choisit pour que $p(s) \neq p\left(s^{\prime}\right)$ si $s \neq s^{\prime}$ et pour que $\left\{a_{j}\right\} \subset \mathcal{P}_{n}$.

Pour chaque $n$, on considère l'empilement $\mathcal{E}_{n}=\mathcal{E}\left(\mathcal{T}_{n}\right)$ donné par le Théorème 1.1 tel que le centre du disque associé à $p^{-1}\left(a_{1}\right)$ soit 0 , celui associé à $p^{-1}\left(a_{2}\right)$ soit 1 et celui associé à $p^{-1}\left(a_{3}\right)$ soit le point à l'infini.

On définit l'application $\phi_{n}: \mathcal{P}_{n} \rightarrow \widehat{\mathbb{C}}$ qui à $p(s)$ associe le centre $c(s)$ du cercle correspondant.

Théorème 3.4. Si la suite de toitures $\left(\mathcal{S}_{n}\right)$ est de valence bornée et séparante alors on peut extraire une sous-suite $\left(n_{k}\right)$ telle que $\left(\phi_{n_{k}}\right)$ converge uniformément vers un homéomorphisme $\phi: X \rightarrow \widehat{\mathbb{C}}$ tel que, pour tout anneau $A \subset X$,

$$
\bmod _{\text {sup }}\left(A, \mathcal{S}_{n_{k}}\right) \asymp \bmod \phi(A) \asymp \bmod _{\text {inf }}\left(A, \mathcal{S}_{n_{k}}\right)
$$

pour $k$ assez grand. Autrement dit, la sous-suite est conforme.

Dans la suite, il sera pratique d'étendre $\phi_{n}$ aux parties de $X$ : étant donné un ensemble $E \subset X$, on peut définir $\phi_{n}(E)$ comme la réunion des tuiles du pavage associé à $\mathcal{E}_{n}$ qui correspondent à $\mathcal{S}_{n}(E)$. On définit de manière similaire $\phi_{n}^{-1}$. Notons que, $\mathcal{S}_{n}$ étant une toiture et $\mathcal{T}_{n} \subset \mathcal{N}\left(\mathcal{S}_{n}\right)$, $\phi_{n}^{-1}$ transforme continua en continua. Quant à $\phi_{n}$, on s'appuiera sur la Proposition 2.3 (2).

La traduction de la séparation se fera à l'aide du Corollaire 3.8 qui suit. 
Le fait de travailler avec de véritables disques peut en fait être allégé. En général, le point important est de pouvoir contrôler leur masse par leur diamètre, ce qui nous motive à donner la définition suivante.

Définition 3.5 (Rondeur). Si $U \subset X$ est un ensemble borné et $x \in U$, alors on définit la rondeur de $U$ par rapport à $x$ comme

$$
\text { Rond }(U, x)=\inf \{R / r, B(x, r) \subset U \subset B(x, R)\} \text {. }
$$

Par Rond $(U)$, on entendra la plus petite valeur de Rond $(U, x)$ quand $x$ parcourt $U$. De plus, si $K \geq 1$, on dira que $U$ est $K$-rond si $\operatorname{Rond}(U) \leq$ $K$.

Pavage associé à un empilement de cercles. Si $D_{1}, D_{2}, D_{3}$ sont trois disques fermés d'intérieurs deux à deux disjoints de $\mathbb{D}$ et sont tangents deux à deux, on désigne par $\Omega$ une composante du complémentaire de ces disques bordés par trois arcs de cercles que l'on appellera interstice. Cet ouvert $\Omega$ est hyperbolique avec trois points marqués, et il existe un unique "centre" i.e., un point $c$ telle que la mesure harmonique de chaque arête vue de $c$ est exactement $1 / 3$. Découpons $\Omega$ en trois parties en considérant les rayons géodésiques issus de $c$ qui aboutissent à chaque point marqué. On associe alors aux disques la partie qui lui est la plus proche. On définit alors $\widehat{D}_{j}=s_{j}$ comme la réunion de $D_{j}$ avec les portions rajoutées par le processus précédent à chaque point de tangence. Les rayons hyperboliques bordant $\widehat{D}_{j}$ forment ses côtés.

Si $\mathcal{E}$ est un empilement de cercles de $\widehat{\mathbb{C}}$ de diamètre plus petit que $\pi$, alors on peut associer un pavage $\mathcal{S}=\mathcal{S}(\mathcal{E})$ comme ci-dessus, et étudier les modules combinatoires associés. Le point crucial de cette construction est que Rond $\left(\widehat{D}_{j}, c_{j}\right) \leq K$ où $c_{j}$ est le centre du disque $D_{j}$ et $K$ est une constante universelle (par le théorème de distorsion de Koebe). Du coup $\mathcal{S}(\mathcal{E})$ est un quasi-empilement de $\widehat{\mathbb{C}}$ au sens de la définition B.1. On dira par la suite que $\mathcal{S}(\mathcal{E})$ est la toiture associée à l'empilement de cercles $\mathcal{E}$.

Dans le cadre des pavages associés à des empilements de cercles, les différentes notions de modules combinatoires coïncident.

Définition 3.6 (anneau empilé). Soit $\mathcal{S}$ la toiture associée à un empilement de cercles de $\widehat{\mathbb{C}}$. Un anneau $A$ est dit empilé s'il est réunion de tuiles de $\mathcal{S}$ et si chaque composante du complémentaire contient au moins un disque de l'empilement.

Dans le cas qui nous intéresse, le Lemme B.3 s'énonce ainsi. 
Lemme 3.7 (du pont). Soient $\mathcal{S}$ la toiture associée à un empilement de cercles $\mathcal{E}$ de $\widehat{\mathbb{C}}$, et $\Gamma$ une famille de courbes. Si pour tout disque $D$ de $\mathcal{E}$ et toute courbe $\gamma \in \Gamma$ qui traverse sa tuile associée $\widehat{D}$, on a $\ell(\gamma \cap 2 K D) \geq \kappa$ diam $D$, où $K$ est la rondeur de la toiture associée, alors il existe une constante $C=C(\kappa)>1$ telle que

$$
\bmod _{2} \Gamma \leq C \cdot \bmod _{2}(\Gamma, \mathcal{S}) .
$$

On en déduit alors une version non-asymptotique de la Proposition B.2 :

Corollaire 3.8. Si $\mathcal{E}$ est un empilement normalisé de valence bornée et si $A$ est un anneau empilé alors

$$
\bmod _{\text {sup }}(A, \mathcal{S}) \asymp \bmod A \asymp \bmod _{\text {inf }}(A, \mathcal{S}),
$$

où les constantes implicites ne dépendent que de la valence.

DÉmonstration. On considère les familles de courbes $\Gamma_{t}$ et $\Gamma_{s}$ qui relient et séparent les composantes de bord. Pour appliquer le Lemme 3.7 , il suffit de montrer l'existence d'une constante $\kappa>0$ qui ne dépend que de la valence telle que si $\gamma$ est l'une de ces courbes qui traverse une pièce $s$, alors diam $\gamma \geq \kappa \operatorname{diam} s$.

Si $\gamma \in \Gamma_{s}$, alors $\gamma$ doit traverser la réunion de ses voisines et contourner au moins un disque ; or le lemme du collier montre que ces pièces ont toute une taille comparable, ce qui montre l'existence de $\kappa>0$ dans ce cas.

Si $\gamma \in \Gamma_{t}$, alors $\gamma$ doit être de diamètre au moins celui d'un côté de $s$ ou de ses voisines. Comme les rayons des cercles bordant un interstice sont comparables, un argument de compacité montre que le diamètre des côtés est aussi comparable à ces rayons.

Donc les hypothèses du Lemme 3.7 sont satisfaites pour un $\kappa>0$ qui ne dépend que de la valence. De plus, le Lemme 2.14 affirme que $\bmod _{\text {inf }} \leq \bmod _{\text {sup }}$, donc on obtient

$$
\bmod _{\text {sup }}(A, \mathcal{S}) \lesssim \bmod A \lesssim \bmod _{\text {inf }}(A, \mathcal{S}) \leq \bmod _{\text {sup }}(A, \mathcal{S})
$$

DÉmonstration du ThÉorÈme 3.4. On suppose que $\left(\mathcal{S}_{n}\right)$ est séparante et de valence bornée. Soit $A \subset X$ un anneau dont les composantes du complémentaire sont $E$ et $F$. Pour $n$ assez grand, on associe le condensateur $\left(\widehat{E}_{n}, \widehat{F}_{n}\right)$ dans $\mathcal{T}_{n}$ par la Proposition $2.3(2)$, et l'anneau empilé $A_{n}$ qui lui correspond sur $\widehat{\mathbb{C}}$. On a donc par les Propositions 2.11 et 2.3 donc

$\bmod _{\text {sup }}\left(A, \mathcal{S}_{n}\right) \asymp \widehat{\bmod }_{\text {sup }}\left(A, \mathcal{S}_{n}\right) \asymp \widehat{\bmod }_{\text {sup }}\left(A_{n}, \mathcal{S}\left(\mathcal{E}\left(\mathcal{T}_{n}\right)\right)\right)=\bmod _{\text {sup }}\left(A_{n}, \mathcal{S}\left(\mathcal{E}\left(\mathcal{T}_{n}\right)\right)\right)$ 
On obtient aussi $\bmod _{\text {inf }}\left(A_{n}, \mathcal{S}\left(\mathcal{E}\left(\mathcal{T}_{n}\right)\right)\right) \lesssim \bmod _{\text {inf }}\left(A, \mathcal{S}_{n}\right)$, car on peut associer à une courbe $\gamma$ de $\Gamma_{s}\left(A_{n}\right)$ une courbe de $\Gamma_{s}(A)$ dont le support est dans le 1-voisinage de $\gamma$ dans $\mathcal{N}\left(\mathcal{S}_{n}\right)$, cf. la démonstration de la Proposition 2.11.

Montrons d'abord que la suite $\left(\phi_{n}\right)_{n}$ est équicontinue en chaque point. On se fixe $\varepsilon>0$ et $m>0$, et on considère $x \in X$. On peut supposer que $x$ est différent de $\left\{a_{1}, a_{2}\right\}$. Soit alors $V$ un voisinage de $x$ disjoint de $\left\{a_{1}, a_{2}\right\}$. Comme l'approximation est séparante, il existe un anneau $A \subset V$ (bordé par des courbes de Jordan) qui sépare $x$ de $X \backslash V$ tel que, pour $n$ assez grand, on ait $\widehat{\bmod }_{\text {sup }}\left(A_{n}, \mathcal{T}_{n}\right) \geq m$, où $A_{n}$ est l'anneau empilé associé. Le Corollaire 3.8 implique

$$
\bmod A_{n} \gtrsim \widehat{\bmod }_{\text {sup }}\left(A_{n}, \mathcal{T}_{n}\right) \geq m
$$

Or pour $n$ assez grand, une des composantes connexes de $\widehat{\mathbb{C}} \backslash A_{n}$ contient $\{0,1\}$, donc le diamètre sphérique de l'autre composante est borné par une constante $C(m)$ qui tend vers 0 quand $m \rightarrow \infty$. On se fixe $m$ assez grand pour que $C(m) \leq \varepsilon$.

Comme la maille des $\left(\mathcal{S}_{n}\right)$ tend vers 0 , il existe un voisinage $W \subset V$ de $x$ disjoint de tous les $A_{n}$ et séparé de $\left\{a_{1}, a_{2}\right\}$. Du coup, pour tout $n$ assez grand, pour tous $s, s^{\prime} \subset W$, on a $d\left(\phi_{n}(p(s)), \phi_{n}\left(p\left(s^{\prime}\right)\right)\right) \leq C(m) \leq$ $\varepsilon$. Ceci implique que la suite $\left(\phi_{n}\right)$ est équicontinue, donc on peut extraire une sous-suite $\left(\phi_{n_{k}}\right)_{k}$ convergente de limite une application continue $\phi: X \rightarrow \widehat{\mathbb{C}}$. Nous renvoyons à l'Appendice $\mathrm{C}$ pour plus de détails sur cette convergence.

On procède en deux étapes pour montrer que $\phi$ est injective. Tout d'abord, si $x \notin\left\{a_{j}\right\}$, alors on peut trouver un anneau $A$ bordé par des courbes de Jordan qui sépare $\left\{x, a_{1}\right\}$ de $\left\{a_{2}, a_{3}\right\}$ de module combinatoire uniformément minoré. Par le Corollaire 3.8, on en déduit que les modules analytiques des anneaux empilés associés sont aussi uniformément minorés, donc $\phi(x) \notin\{1, \infty\}$, sinon le module devrait tendre vers zéro. Par permutation cyclique, on trouve que $\phi(x) \notin$ $\{0,1, \infty\}$.

Dans un second temps, on considère $x \neq y$. On peut supposer que $x, y, a_{1}, a_{2}$ sont quatre points distincts. En considérant un anneau qui sépare $\left\{x, a_{1}\right\}$ de $\left\{y, a_{2}\right\}$, on montre que $\phi(x) \neq \phi(y)$ par le même argument.

Par conséquent, $\phi$ est un homéomorphisme de $X$ qui est une sphère topologique sur son image. On en déduit que $\phi: X \rightarrow \widehat{\mathbb{C}}$ est un homéomorphisme. 
La comparaison des différents modules associés à un anneau suit aussi du Corollaire 3.8 (voir aussi le Théorème 7.1 dans [8]). En effet, si $A \subset \widehat{\mathbb{C}}$ et $\left(\mathcal{E}_{n_{k}}\right)$ est la suite d'empilements associée à $\left(\mathcal{T}_{n_{k}}\right)$, alors $\left(\mathcal{S}\left(\mathcal{E}_{n_{k}}\right)\right)$ est conforme. En effet, si $A \subset \widehat{\mathbb{C}}$ est un anneau bordé par des courbes de Jordan, on note $A_{k}$ l'anneau empilé associé à $\mathcal{E}_{n_{k}}$. Comme la maille des recouvrements $\mathcal{S}_{n}$ tend vers 0 et puisque la suite $\left(\phi_{n_{k}}\right)$ est convergente, on en déduit que la maille des $\left(\mathcal{S}\left(\mathcal{E}_{n_{k}}\right)\right)$ tend aussi vers 0 . Du coup, $\bmod A_{k} \rightarrow \bmod A$, et les hypothèses du Corollaire 3.8 sont vérifiées pour $\Gamma_{s}\left(A_{k}\right)$ et $\Gamma_{t}\left(A_{k}\right)$. Donc, pour $k$ assez grand, on obtient ainsi

$$
\bmod _{\text {sup }}\left(A, \mathcal{S}\left(\mathcal{E}_{n_{k}}\right)\right) \asymp \bmod _{\text {inf }}\left(A, \mathcal{S}\left(\mathcal{E}_{n_{k}}\right)\right) \asymp \bmod A_{k} \asymp \bmod A .
$$

Considérons maintenant un anneau $A \subset X$ bordé par des courbes de Jordan. L'anneau $\phi(A)$ s'obtient donc comme limite d'anneaux empilés $A_{k}$ donc $\bmod A_{k}$ tend vers $\bmod \phi(A)$. Du coup, puisque la maille des empilements tend vers 0 ,

$$
\widehat{\bmod }_{\text {sup }}\left(A, \mathcal{S}_{n_{k}}\right) \asymp \widehat{\bmod }_{\text {sup }}\left(\phi_{n_{k}}(A), \mathcal{S}\left(\mathcal{E}_{n_{k}}\right)\right) \asymp \bmod \phi(A)
$$

pour $k$ assez grand. La Proposition 2.11 montre que $\bmod _{\text {sup }}\left(A, \mathcal{S}_{n_{k}}\right) \asymp$ $\bmod \phi(A), c f$. (3.1). Par ailleurs,

$$
\bmod _{\text {sup }}\left(A, \mathcal{S}_{n_{k}}\right) \geq \bmod _{\text {inf }}\left(A, \mathcal{S}_{n_{k}}\right) \gtrsim \bmod _{\text {inf }}\left(A_{n_{k}}, \mathcal{T}_{n_{k}}\right) \asymp \bmod \phi(A) .
$$

Remarque 3.9. L'équicontinuité de $\left(\phi_{n}\right)_{n}$ établie dans la démonstration montre notamment que, pour tout anneau $A$, il existe une constante $K=K(A)$ telle que les modules combinatoires de $A$ soient $K$-comparables entre eux. On peut se demander si cela n'implique pas que la suite soit automatiquement conforme : la réponse est négative. En effet, soit $\left(\mathcal{E}_{n}\right)_{n}$ une suite d'empilements de valence bornée dont la maille tend vers 0 et de pavage associé $\left(\mathcal{P}_{n}\right)$; considérons aussi un homéomorphisme $h: \widehat{\mathbb{C}} \rightarrow \widehat{\mathbb{C}}$ non quasiconforme, et posons $\mathcal{S}_{2 n}=\mathcal{P}_{n}$ et $\mathcal{S}_{2 n+1}=h^{-1}\left(\mathcal{P}_{n}\right)$. La suite $\left(\mathcal{S}_{n}\right)$ est bien séparante, mais elle n'est pas conforme puisque $h$ n'est pas quasiconforme.

Ceci montre que sous nos hypothèses, deux structures complexes construites par le théorème ne sont pas forcément dans la même classe quasiconforme (si $\Phi$ et $\Psi$ sont deux homéomorphismes limites, $\Phi \circ \Psi^{-1}$ n'est pas forcément quasiconforme). Une modification de l'exemple cidessus permet aussi de montrer que le fait que toute limite est dans la même classe quasiconforme n'implique pas que la suite est conforme: considérons maintenant une suite d'homéomorphismes quasiconformes 
$\left(h_{k}\right)$ qui tend uniformément vers l'identité avec une distorsion qui diverge. On construit une suite de pavages en utilisant $h_{k}^{-1}\left(\mathcal{P}_{n}\right), k, n \in$ $\mathbb{N}$ : cette suite sera bien séparante, les homéomorphismes limites seront les $h_{k}$ ou l'identité, mais la suite n'est pas conforme car la suite $\left(h_{k}\right)$ n'est pas uniformément quasiconforme.

On en déduit une version faible du théorème de Riemann combinatoire.

Corollaire 3.10. Si la suite $\left(\mathcal{S}_{n}\right)$ est une suite de toitures de valence bornée et conforme, chaque $\mathcal{S}_{n}$ contenant une triangulation équivalente $\mathcal{T}_{n}$, alors on peut extraire une sous-suite $\left(n_{k}\right)$ telle que $\left(\phi_{n_{k}}\right)$ converge uniformément vers un homéomorphisme $\phi: X \rightarrow \widehat{\mathbb{C}}$ tel que

$$
\bmod _{\text {sup }}\left(A, \mathcal{S}_{n}\right) \asymp \bmod \phi(A) \asymp \bmod _{\inf }\left(A, \mathcal{S}_{n}\right)
$$

pour $n$ assez grand. De plus, si $\phi$ et $\psi$ sont deux homéomorphismes limites, alors $\phi \circ \psi^{-1}$ est $K$-quasiconforme où $K$ ne dépend que des données.

Réciproquement, si $\left(\phi_{n}\right)$ est convergente vers un homéomorphisme alors $\left(\mathcal{S}_{n}\right)$ est conforme.

DÉmonstration. Le Théorème 3.4 s'applique pour montrer la convergence d'une sous-suite $\left(\phi_{n_{k}}\right)_{k}$ vers un homéomorphisme $\phi$. Conjugué à la conformité de la suite, ce théorème fournit aussi les estimations entre les modules, et permet de montrer que si $\phi$ et $\psi$ sont deux limites, alors $\phi \circ \psi^{-1}$ préserve les modules à une constante multiplicative uniforme près, autrement dit $\phi \circ \psi^{-1}$ est $K$-quasiconforme où $K$ ne dépend que des données ( $c f$. appendice $\mathrm{A}$ ).

Quant à la réciproque, elle découle du même argument que dans la démonstration du Théorème 3.4.

3.2. Paramétrage quasisymétrique de sphères topologiques. Dans ce paragraphe, on exhibe des conditions (analytiques) sur une sphère topologique qui permettent de conclure qu'elle est quasisymétrique à la sphère de Riemann. Notre point de départ sera toujours la donnée d'une suite de quasi-empilements par tuiles de valence bornée. Nous verrons comment, avec l'approche du paragraphe précédent, établir des résultats de $\mathrm{M}$. Bonk et $\mathrm{B}$. Kleiner.

On se réfère de manière essentielle à l'appendice A pour les définitions, les notations et les propriétés des objets analytiques utilisées dans ce paragraphe.

Le premier résultat nous servira pour conclure que les limites des $\left(\phi_{n}\right)$ construites au paragraphe précédent sont quasisymétriques. 
Proposition 3.11. Soient $X$ un espace doublant et $\left(\mathcal{S}_{n}\right)$ une suite de quasi-empilements qui vérifie les hypothèses du Théorème 3.4. Les propositions suivantes sont équivalentes.

(1) Il existe $\eta$ telle que tout homéomorphisme limite $\phi$ est $\eta$-quasisymétrique.

(2) Il existe un homéomorphisme $\psi_{1}: \mathbb{R}_{+} \backslash\{0\} \rightarrow \mathbb{R}_{+} \backslash\{0\}$ décroissant tel que pour tout condensateur $(E, F)$, on ait $\bmod _{2}\left(E, F, \mathcal{S}_{n}\right) \geq$ $\psi_{1}(\Delta(E, F))$ pour tout $n$ assez grand.

(3) L'espace $X$ est linéairement localement connexe et il existe un homéomorphisme $\psi_{2}: \mathbb{R}_{+} \backslash\{0\} \rightarrow \mathbb{R}_{+} \backslash\{0\}$ décroissant tel que, pour tout condensateur $(E, F)$, on a $\bmod _{2}\left(E, F, \mathcal{S}_{n}\right) \leq$ $\psi_{2}(\Delta(E, F))$ pour tout $n$ assez grand.

De plus, la suite $\left(\mathcal{S}_{n}\right)_{n}$ est conforme.

DÉmonstration. Une application quasisymétrique contrôle le rapport de distances, en particulier les distances relatives $\Delta(E, F)$ ( $c f$. Lemme 3.2 dans [5]). Donc si $\phi$ est $\eta$-quasisymétrique, alors il existe des homéomorphismes $\chi_{1}, \chi_{2}: \mathbb{R}_{+} \rightarrow \mathbb{R}_{+}$ne dépendant que de $\eta$ tels que

$$
\chi_{1}(\Delta(E, F)) \geq \Delta(\phi E, \phi F) \geq \chi_{2}(\Delta(E, F)) .
$$

Sur $\widehat{\mathbb{C}}$, il existe des homéomorphismes $\widehat{\psi}_{1}, \widehat{\psi}_{2}: \mathbb{R}_{+} \backslash\{0\} \rightarrow \mathbb{R}_{+} \backslash\{0\}$ décroisssantes vers 0 telles que

$$
\widehat{\psi}_{2}(\Delta(E, F)) \geq \bmod _{2}(E, F) \geq \widehat{\psi}_{1}(\Delta(E, F)) .
$$

On se fixe $(E, F)$ et on définit

$$
m_{s}=\limsup \bmod _{2}\left(E, F, \mathcal{S}_{n}\right) \quad \text { et } \quad m_{i}=\liminf \bmod _{2}\left(E, F, \mathcal{S}_{n}\right) \text {. }
$$

Montrons que

$$
m_{s} \lesssim m_{i}
$$

où les constantes implicites ne dépendent pas du condensateur. On se donne les sous-suites $\left(\mathcal{S}_{n_{k}, s}\right)$ et $\left(\mathcal{S}_{n_{k}, i}\right)$ qui font tendre les modules vers $m_{s}$ et $m_{i}$. Le Théorème 3.4 produit deux homéomorphismes $\eta$ quasisymétriques $\phi_{s}, \phi_{i}: X \rightarrow \widehat{\mathbb{C}}$ tels que

$$
m_{s} \asymp \bmod _{2}\left(E, F, \mathcal{S}_{n_{k}, s}\right) \asymp \bmod _{2}\left(\phi_{s}(E), \phi_{s}(F)\right)
$$

et

$$
m_{i} \asymp \bmod _{2}\left(E, F, \mathcal{S}_{n_{k}, i}\right) \asymp \bmod _{2}\left(\phi_{i}(E), \phi_{i}(F)\right) .
$$

Or $\phi_{s} \circ \phi_{i}^{-1}$ est un homéomorphisme $\widehat{\eta}$-quasisymétrique de la sphère de Riemann, où $\widehat{\eta}(t)=\eta\left(1 / \eta^{-1}(1 / t)\right)$. Donc $\phi_{s} \circ \phi_{i}^{-1}$ quasipréserve les 2-modules. Par conséquent, $m_{s} \lesssim m_{i}$ établissant (3.2).

Cela signifie qu'il suffit de travailler avec une sous-suite $\left(\mathcal{S}_{n_{k}}\right)$ pour laquelle on a convergence des injections vers un homéomorphisme $\phi$. 
D'après le Théorème 3.4,

$$
\bmod _{2}\left(E, F, \mathcal{S}_{n_{k}}\right) \asymp \bmod _{2}(\phi E, \phi F)
$$

pour $k$ assez grand, donc, pour $n$ assez grand et en vertu de (3.2)

$$
\left\{\begin{array}{l}
\bmod _{2}\left(E, F, \mathcal{S}_{n}\right) \asymp \bmod _{2}(\phi E, \phi F) \geq \widehat{\psi}_{1}(\Delta(\phi E, \phi F)) \geq \widehat{\psi}_{1} \chi_{1}(\Delta(E, F)) \\
\bmod _{2}\left(E, F, \mathcal{S}_{n}\right) \asymp \bmod _{2}(\phi E, \phi F) \leq \widehat{\psi}_{2}(\Delta(\phi E, \phi F)) \leq \widehat{\psi}_{2} \chi_{2}(\Delta(E, F)),
\end{array}\right.
$$

ce qui montre l'existence des applications $\psi_{1}$ et $\psi_{2}$ sous la condition que $\phi$ est quasisymétrique. Notons que la sphère est linéairement localement connexe, et cette propriété est préservée par applications quasisymétriques (cf. [14, Chap. 15]).

Pour la réciproque, on note que $X$ étant doublant, il suffit de montrer que toute limite $\phi$ est uniformément faiblement quasisymétrique (voir la fin de l'appendice $\S \mathrm{A}$, ou [14, Thm 10.19]). On se donne $x \in X$ et $r>0$ et on note $L=L_{\phi}(x, r), \ell=\ell_{\phi}(x, r), A=D(\phi(x), L) \backslash D(\phi(x), \ell)$, où $D(z, r) \subset \widehat{\mathbb{C}}$ est le disque centré en $z$ de rayon $r$ pour la métrique sphérique, et $\Gamma_{t}$ la famille des courbes qui joignent les deux composantes de bord de $A$. Si $L / \ell \leq 2$, alors, on n'a rien à montrer. Sinon, on remarque que $\Delta(A) \asymp(L-\ell) / \ell \asymp L / \ell$, où $\Delta(A)$ désigne la distance relative des deux composantes de bord de $A$.

D'une part, on a

$$
0<c \leq \psi_{1}\left(\Delta\left(\phi^{-1}(A)\right)\right) \leq \bmod _{2}\left(\Gamma_{t}, \mathcal{S}_{n}\right)
$$

par hypothèses, et d'autre part, si $n$ est assez grand,

$$
\bmod _{2}\left(\Gamma_{t}, \mathcal{S}_{n}\right) \lesssim 1 / \log L / \ell .
$$

En effet, il suffit de considérer les poids $\rho_{n}(s)=\operatorname{diam} \phi_{n}(s) / \operatorname{dist}\left(\phi(x), \phi_{n}(s)\right)$ pour $s \in \mathcal{S}_{n}(A)$ et $\rho_{n}(s)=0$ sinon ( $c f$. Lemme A.8, et la démonstration du Corollaire 3.14). Donc ces deux inégalités montrent que le rapport $L / \ell$ est borné indépendamment de $\phi, x$ et $r>0$, donc $\phi$ est uniformément faiblement quasisymétrique.

Quant à l'autre condition, (3), on montre que $\phi^{-1}$ est quasisymétrique en inversant les rôles. Soit $C$ la constante de connexité locale linéaire de $X$. Soient $z, z_{1}, z_{2} \in \widehat{\mathbb{C}}$ tels que $\left|z-z_{1}\right| \leq\left|z-z_{2}\right|$. Si $\left|\phi^{-1}(z)-\phi^{-1}\left(z_{1}\right)\right| \leq$ $2 C^{2}\left|\phi^{-1}(z)-\phi^{-1}\left(z_{2}\right)\right|$, nous n'avons rien à montrer. Sinon, on peut supposer que $z, z_{1}, z_{2}$ sont loin de l'infini et, par continuité uniforme que leurs images par $\phi^{-1}$ sont loin de $a_{3}$. On construit deux continua disjoints $E$ et $F$ sur $X$ tels que

$$
\phi^{-1}(z), \phi^{-1}\left(z_{2}\right) \in E \subset B\left(\phi^{-1}(z), C\left|\phi^{-1}(z)-\phi^{-1}\left(z_{2}\right)\right|\right)
$$

et

$$
\phi^{-1}\left(z_{1}\right), a_{3} \in F \subset X \backslash B\left(\phi^{-1}(z),(1 / C)\left|\phi^{-1}(z)-\phi^{-1}\left(z_{1}\right)\right|\right) .
$$


On a donc

$$
\Delta(E, F) \geq\left(1 / C^{3}\right) \frac{\left|\phi^{-1}(z)-\phi^{-1}\left(z_{1}\right)\right|}{\left|\phi^{-1}(z)-\phi^{-1}\left(z_{2}\right)\right|}
$$

et $\Delta(\phi(E), \phi(F)) \leq 1$. Par suite,

$$
0<\widehat{\psi}_{1}(1) \lesssim \widehat{\bmod }_{2}\left(E, F, \mathcal{S}_{n}\right) \leq \psi_{2}\left(\left(1 / C^{3}\right) \frac{\left|\phi^{-1}(z)-\phi^{-1}\left(z_{1}\right)\right|}{\left|\phi^{-1}(z)-\phi^{-1}\left(z_{2}\right)\right|}\right) .
$$

Comme $\psi_{2}$ est décroissante, on en déduit que $\left|\phi^{-1}(z)-\phi^{-1}\left(z_{1}\right)\right| \lesssim$ $\left|\phi^{-1}(z)-\phi^{-1}\left(z_{2}\right)\right|$, et donc que $\phi^{-1}$ est quasisymétrique.

L'estimation (3.2) s'interprète par la conformité de $\left(\mathcal{S}_{n}\right)$ grâce à la conclusion du Théorème 3.4 sur les estimations des modules.

Remarque 3.12. Dans [5], M. Bonk et B. Kleiner travaillent avec ce qu'ils appellent des $K$-approximations $\left(\mathcal{A}_{n}\right)$ qui ne sont pas des toitures (voir § 4). Cependant, ce sont des quasi-empilements de valence bornée qui ont des propriétés supplémentaires à nos suites de recouvrements ; notamment, deux points d'une même pièce $U \in \mathcal{A}_{n}$ peuvent être joints par une courbe dont le support est contenu dans un $K$-voisinage de $U$ dans le nerf de $\mathcal{A}_{n}$ et de diamètre comparable à celui de $U$. Donc, en agrandissant les pièces de $\mathcal{A}_{n}$, on obtient un quasi-empilement par tuiles $\mathcal{S}_{n}$ (a) de valence bornée dont le nerf est équivalent à celui de $\mathcal{A}_{n}$ au sens de la définition 2.4 et (b) d'indice de recouvrement fini. Par conséquent, les Propositions 2.3 et 2.11, et le Lemme 2.9 impliquent que les modules combinatoires $\widehat{\bmod }_{*}$ et $\bmod _{*}$ sont tous comparables, et nous restons bien dans le cadre qu'ils traitent.

Notons que la construction de ces recouvrements est délicate et repose sur le fait que $X$ est doublant et linéairement localement connexe. M. Bonk et B. Kleiner définissent leurs approximations en construisant une triangulation plongée dans $X$, voir $[5, \S 6]$.

Nous pouvons directement en déduire ce que M. Bonk et B. Kleiner appellent le résultat principal de leur article [5] (Théorème 11.1) :

Corollaire 3.13 (M. Bonk \& B. Kleiner). Soit X un espace métrique doublant et linéairement localement connexe homéomorphe à $\mathbb{S}^{2}$. On suppose que l'on a une suite $\left(\mathcal{S}_{n}\right)$ de quasi-empilements par tuiles dont la maille tend vers zéro et dont les nerfs contiennent des triangulations équivalentes de la sphère de valence bornée.

$S$ 'il existe des constantes $\lambda>1$ et $C>0$ telles que

$$
\widehat{\bmod }_{2}\left(B, X \backslash \lambda B, \mathcal{S}_{n}\right) \leq C
$$

pour toute boule $B \subset X$ et pour $n$ assez grand, alors $X$ est quasisymétrique à $\widehat{\mathbb{C}}$ et $\left(\mathcal{S}_{n}\right)$ est conforme. 
Démonstration. Soient $x \in X$ et $r>0$ et considérons, comme M. Bonk et B. Kleiner, les anneaux $B\left(x, \lambda^{-2 j} r\right) \backslash B\left(x, \lambda^{-(2 j+1)} r\right)$. Par l'inégalité de Grötzsch [2, Thm 4.2] et nos hypothèses, on obtient pour $n$ assez grand

$\widehat{\bmod }_{\text {sup }}\left(B(x, r) \backslash B\left(x, \lambda^{-(2 k+1)} r\right), \mathcal{S}_{n}\right) \geq \sum_{j=0}^{k} \widehat{\bmod }_{\text {sup }}\left(B\left(x, \lambda^{-2 j} r\right) \backslash B\left(x, \lambda^{-(2 j+1)} r\right), \mathcal{S}_{n}\right) \geq k / C$.

Donc $\left(\mathcal{S}_{n}\right)$ vérifie la condition (2) d'approximation séparante qui permet de montrer que la suite $\left(\phi_{n}\right)$ est équicontinue. S'il y avait une fibre non triviale $F$, alors on pourrait trouver une boule $B \subset X$ telle que les diamètres de $\phi(B)$ et de $\phi(X \backslash \lambda B)$ sont minorés, avec les intersections $B \cap F$ et $(X \backslash \lambda B) \cap F$ non vides, donc telle que $\phi(B) \cap \phi(X \backslash \lambda B) \neq \emptyset$. Par le Corollaire 3.8, ceci contredit $\widehat{\bmod }_{2}\left(B, X \backslash \lambda B, \mathcal{S}_{n}\right) \leq C$ pour $n$ assez grand. Donc les conclusions du Théorème 3.4 sont vérifiées.

La Proposition 3.11 (3) s'applique, ce qui montre que la limite $\phi$ est quasisymétrique et $\left(\mathcal{S}_{n}\right)$ est conforme.

On en déduit ( $c f$. Théorème 10.4 de [5]) :

Corollaire 3.14. Si $(X, \mu)$ est un espace métrique 2 -Ahlfors régulier linéairement localement connexe homéomorphe à $\mathbb{S}^{2}$, et si $\left(\mathcal{S}_{n}\right)$ est une suite de quasi-empilements par tuiles de valence bornée dont le nerf contient une triangulation équivalente et la maille tend vers 0 alors $\left(\mathcal{S}_{n}\right)$ est conforme et $X$ est quasisymétrique à $\widehat{\mathbb{C}}$.

DÉmonstration. Il suffit de vérifier que les hypothèses du Corollaire 3.13 sont vérifiées. On se fixe $x \in X$, et $r<R=2^{k} r<\operatorname{diam} X$, où $k \geq 1$. On définit la métrique $\rho_{n}: \mathcal{S}_{n}(B(x, R) \backslash B(x, r)) \rightarrow \mathbb{R}_{+}$ par $\rho_{n}(s)=\operatorname{diam} s / \operatorname{dist}(x, s)(c f$. Lemme A.8). Alors, en découpant en couronnes diadiques centrées en $x$, pour toute $\gamma \in \widehat{\Gamma}_{t}$, on a

$$
\ell_{\rho_{n}}(\gamma) \geq(1 / r) \sum_{j=0}^{k-1} 2^{-j} \sum \operatorname{diam} s \gtrsim \sum_{j=1}^{k} 2^{-j} 2^{j} \gtrsim k \gtrsim \log R / r
$$


De même, en utilisant la régularité de $X$, les propriétés de rondeur et de valence bornée, on a

$$
\begin{aligned}
\sum \rho_{n}(s)^{2} & \lesssim \sum_{j=0}^{k-1} \sum \frac{(\operatorname{diam} s)^{2}}{\left(2^{j} r\right)^{2}} \\
& \lesssim \sum_{j=0}^{k-1} \frac{\mu\left(B\left(x, 2^{j+1} r\right)\right)}{\left(2^{j} r\right)^{2}} \\
& \lesssim \sum_{j=0}^{k-1} \frac{\left(2^{j+1} r\right)^{2}}{\left(2^{j} r\right)^{2}} \\
& \lesssim \log R / r .
\end{aligned}
$$

Donc $\widehat{\bmod }_{2}\left(B(x, r), X \backslash B(x, R), \mathcal{S}_{n}\right) \lesssim 1 / \log R / r$, et le Corollaire 3.13 s'applique.

On peut aussi retrouver une version asymptotique du Théorème 10.1 de M. Bonk et B. Kleiner dans [5] :

Théorème 3.15. Soit $X$ un espace métrique $Q$-Loewner et $Q$-Ahlfors régulier homéomorphe à $\mathbb{S}^{2}$. On suppose que l'on a une suite de quasiempilements par tuiles de valence bornée dont la maille tend vers zéro et dont le nerf contient une triangulation équivalente de la sphère. Alors $\left(\mathcal{S}_{n}\right)$ est conforme, $X$ est quasisymétrique à $\widehat{\mathbb{C}}$ et $Q=2$.

DÉmonstration. La condition loewnesque permet de minorer les $Q$ modules de courbes : elle nous fournit l'existence d'une application décroissante $\psi_{1}$ telle que, pour tout condensateur $(E, F)$ de $X$,

$$
\bmod _{Q}(E, F) \geq \psi_{1}(\Delta(E, F)) \text {. }
$$

Or, $Q \geq 2$ implique que $\bmod _{Q}\left(\Gamma, \mathcal{S}_{n}\right) \leq \bmod _{2}\left(\Gamma, \mathcal{S}_{n}\right)$ en normalisant les métriques par $L_{\rho}(\Gamma)=1$ pour toute famille de courbes ; donc on obtient avec le Lemme B.3

$$
\psi_{1}(\Delta(E, F)) \leq \bmod _{Q}(E, F) \lesssim \bmod _{Q}\left(E, F, \mathcal{S}_{n}\right) \leq \bmod _{2}\left(E, F, \mathcal{S}_{n}\right) .
$$

De plus, le Corollaire 3.8 et le Lemme A.8 impliquent l'existence d'une autre application décroissante $\psi_{2}$ telle que $\bmod _{2}\left(E^{\prime}, F^{\prime}, \mathcal{S}\left(\mathcal{E}_{n}\right)\right) \lesssim \bmod _{2}\left(E^{\prime}, F^{\prime}\right) \leq$ $\psi_{2}\left(\Delta\left(E^{\prime}, F^{\prime}\right)\right)$ pour tout condensateur $\left(E^{\prime}, F^{\prime}\right)$ de $\widehat{\mathbb{C}}$ et $n$ assez grand. En particulier, pour tout condensateur $(E, F)$ de $X$, et tout $n$ assez grand, on a

$$
\psi_{1}(\Delta(E, F)) \lesssim \psi_{2}\left(\Delta\left(\phi_{n} E, \phi_{n} F\right)\right)
$$


Nous allons montrer que la suite $\left(\phi_{n}^{-1}\right)_{n}$ est équicontinue, et que toute limite produit un homéomorphisme de $\widehat{\mathbb{C}}$ sur $X$. Pour cela, on montre d'abord que la maille des empilements de cercles tend vers 0 .

Si ce n'est pas le cas, il existe $r_{0}>0$, une sous-suite $\left(n_{k}\right)$ et un élément $s_{k} \in \mathcal{S}_{n_{k}}$ tels que son disque associé $D_{k}$ est de rayon au moins $r_{0}$. Quitte à extraire une sous-suite, on peut supposer que $\left(s_{k}\right)$ tend vers un point $x \in X$ différent de $a_{1}, a_{2}$. Rappelons que chaque nerf $\mathcal{N}\left(\mathcal{S}_{n}\right)$ contient la triangulation équivalente $\mathcal{T}_{n}$; il existe donc une constante $K$ telle que si $s, s^{\prime} \in \mathcal{S}_{n}$ sont à distance combinatoire au moins $K$ dans $\mathcal{T}_{n}$, alors leur distance est au moins 2 dans $\mathcal{N}\left(\mathcal{S}_{n}\right)$. Soit $\gamma_{k} \subset \widehat{\mathbb{C}}$ un arc qui joint 0 à $D_{k}$; notons $E_{k} \subset \widehat{\mathbb{C}}$ le continuum constitué de $\mathcal{S}_{n_{k}}\left(\gamma_{k}\right)$ et $V_{k}$ la réunion des tuiles qui forment le $K$-voisinage de $E_{k}$ dans la triangulation $\mathcal{T}_{n_{k}}$. Pour $k$ assez grand, $V_{k}$ est bordé par une courbe de Jordan puisque la triangulation est planaire, et quitte à modifier $\gamma_{k}$, on peut aussi supposer que 1 n'y appartient pas. On construit alors un autre continuum $F_{k} \subset \widehat{\mathbb{C}} \backslash V_{k}$ formé de tuiles de $\mathcal{S}\left(\mathcal{E}_{n_{k}}\right)$ qui relie 1 à une pièce $\widehat{D_{k}^{\prime}}=\phi_{n_{k}}\left(s_{k}^{\prime}\right)$, où $s_{k}^{\prime}$ est à distance $2 K$ de $s_{k}$ dans $\mathcal{T}_{n_{k}}$, de sorte que $\Delta\left(E_{k}, F_{k}\right) \geq \delta_{0}>0$, où $\delta_{0}$ est une constante indépendante de $k$; l'existence de $\delta_{0}$ provient du fait que les disques $D_{k}, D_{k}^{\prime}$ associés à $s_{k}$ et $s_{k}^{\prime}$ sont à distance combinatoire uniformément bornée et de rayon minoré, $c f$. le lemme du collier. Or $\Delta\left(\phi_{n_{k}}^{-1}\left(E_{k}\right), \phi_{n_{k}}^{-1}\left(F_{k}\right)\right)$ tend vers 0 , car les diamètres de $\phi_{n_{k}}^{-1}\left(E_{k}\right)$ et $\phi_{n_{k}}^{-1}\left(F_{k}\right)$ sont minorés par $\min \left\{d_{X}\left(a_{1}, x\right), d_{X}\left(a_{2}, x\right)\right\} / 2$, la maille de $\mathcal{S}_{n_{k}}$ tend vers 0 et $s_{k}$ et $s_{k}^{\prime}$ sont à distance combinatoire au plus $2 K$. Ceci contredit (3.3).

Il s'ensuit que la suite d'empilements de cercles $\left(\mathcal{E}_{n}\right)_{n}$ a sa maille qui tend vers 0 , donc le Corollaire 3.8 implique que la suite des toitures associées est conforme. La majoration (3.3) implique facilement l'équicontinuité de $\left(\phi_{n}^{-1}\right)$. Notons que les arguments de la démonstration du Théorème 3.4 permettent de montrer l'injectivité de la limite, ainsi que la conformité de $\left(\mathcal{S}_{n}\right)$. La Proposition 3.11 implique alors que $\phi^{-1}$ est quasisymétrique.

Notons que $\left(\mathcal{S}_{n}\right)$ étant conforme, on a forcément $Q=2$. Pour $Q>2$, on aurait $\lim _{n} \bmod _{Q}\left(\cdot, \mathcal{S}_{n}\right)=0$. En effet, si $(E, F)$ est un condensateur de $X$ et $\phi: X \rightarrow \widehat{\mathbb{C}}$ est l'homéomorphisme donné par le Théorème 3.4 , alors on considère $\rho_{n}(s)=\operatorname{diam} \phi(s)$ pour $s \in \mathcal{S}_{n}$. Il vient, pour $\gamma \in \Gamma(E, F)$

$$
\ell_{\rho_{n}}\left(\gamma, \mathcal{S}_{n}\right)=\sum_{\mathcal{S}_{n}(\gamma)} \operatorname{diam} \phi(s) \geq \operatorname{dist}(\phi(E), \phi(F))
$$


et

$V_{Q, \rho_{n}}(X) \lesssim\left(\sup _{\mathcal{S}\left(\mathcal{E}_{n}\right)} \operatorname{diam} s\right)^{Q-2} \cdot \sum_{s \in \mathcal{S}\left(\mathcal{E}_{n}\right)}(\operatorname{diam} s)^{2} \lesssim\left(\sup _{\mathcal{E}_{n}} \operatorname{diam} s\right)^{Q-2} \operatorname{Aire}(\widehat{\mathbb{C}})$

qui tend vers 0 dès que $Q>2$.

Remarque 3.16. Ce dernier argument montre qu'en fait, si $X$ est $Q$-Ahlfors régulier pour un $Q>2$, et si la suite de quasi-empilements est conforme, alors le $Q$-module de tout condensateur est nul, et les $Q$ modules combinatoires associés tendent vers 0 par la Proposition B.2. Une condition nécessaire pour avoir la conformité d'une suite est donc que le $Q$-module combinatoire de tout condensateur tend vers 0 pour tout $Q>2$.

Modulo la construction des quasi-empilements par tuiles de valence bornée dont le nerf contient une triangulation équivalente ( $c f$. $\S 4$ et $\S 6$ dans [5] et la Remarque 3.12), on récupère ainsi des résultats de M. Bonk et B. Kleiner (Théorème 1.1 et 1.2 de [5]) :

Théorème 3.17 (M. Bonk et B. Kleiner). Si X est homéomorphe à $\mathbb{S}^{2}$, linéairement localement connexe et $Q$-régulier, et si on suppose que, ou bien $Q=2$ ou bien $X$ est $Q$-loewnesque, alors $X$ est quasisymétrique $\grave{a} \widehat{\mathbb{C}}($ et $Q=2)$.

3.3. Remarque sur l'utilisation des toitures. Tous nos résultats sont établis pour des toitures. En fait, cette condition n'est essentiellement utilisée que pour comparer les modules $\widehat{\bmod }_{*}$ et $\bmod _{*}(c f$. (3.1)) et pour obtenir que les suites sont conformes. Si on s'affranchit des toitures, tous les résultats restent vrais en remplaçant $\bmod _{*}$ par $\widehat{\bmod }_{*}$ dans les hypothèses et les conclusions. Seule la démonstration du Théorème 3.15 requiert quelques changements car $\phi_{n}^{-1}$ ne préserve plus les continua a priori; une méthode est de se ramener au Corollaire 3.13 : on considère $x \in X$ et $r<R \leq \operatorname{diam} X$ et on veut montrer qu'il existe une constante $C$ qui ne dépend que de $R / r$ telle que $\widehat{\bmod }_{2}\left(B(x, r), X \backslash B(x, R), \mathcal{S}_{n}\right) \leq C$. On note $E=B(x, r)$ et $F=X \backslash B(x, R)$; si $R / r$ est assez grand, alors la connexité locale linéaire de $X$ loewnesque permet de supposer $E$ et $F$ connexes. En partant de $(3.3)$, on suppose que $\Delta\left(\phi_{n}(E), \phi_{n}(F)\right) \leq \delta$, et l'on veut montrer que $\delta$ ne peut être trop petit en construisant de nouveaux condensateurs qui feront intervenir la condition de Loewner pour minorer $\delta$. On obtiendra ainsi un bon contrôle du 2-module en utilisant que $\widehat{\mathbb{C}}$ est 2-régulier. 


\subsection{Sur les démonstrations des différentes versions du théorème}

de Riemann. Dans tous ces résultats, outre l'hypothèse topologique d'être une sphère, on part d'hypothèses qui imposent la présence de nombreuses courbes sur la surface. Ces courbes sont quantifiées grâce à la notion de modules, et ceux-ci sont utilisés pour en déduire des propriétés géométriques de la surface. On suppose donc toujours une condition géométrico-combinatoire extrême : dans les hypothèses de séparation ou de conformité, on se donne la combinatoire de la sphère de Riemann, dans l'hypothèse 2-Ahlfors régulière, on se donne une borne supérieure sur la géométrie des modules, et sous la condition loewnesque, on obtient une borne inférieure. De plus, dans les deux approches, les auteurs imposent des estimations uniformes qui impliquent que les structures complexes qu'ils définissent sont à distance quasiconforme bornée les unes des autres.

L'approche de J.W. Cannon et al. est combinatoire et locale, celle de M. Bonk et B. Kleiner analytique et globale. Les démonstrations que nous avons présentées s'inspirent des deux écoles et permettent de distinguer deux problèmes différents : le premier est de munir la surface considérée d'une structure complexe compatible avec les modules combinatoires, le second consiste à montrer que la structure métrique ainsi obtenue est quasisymétrique à l'originale (lorsque celle-ci est fixée). Le second problème s'interpète alors comme une quantification/habillage d'une approche plus combinatoire.

Nous sommes donc partis de l'existence d'une suite de recouvrements dont les nerfs sont essentiellement des triangulations et dont l'existence est assurée par M. Bonk et B. Kleiner sous de bonnes conditions (bien que nous n'ayons pas besoin de toutes leurs propriétés, il n'est pas clair comment simplifier leur construction).

L'objet du Théorème 3.4 dont les hypothèses de séparation sont inspirées des travaux de J.W. Cannon et al. [10] est de répondre au premier problème par un théorème de Riemann combinatoire à la Cannon. Pour cela, on s'appuie sur la théorie des empilements de cercles, introduite dans ce contexte par M. Bonk et B. Kleiner, qui nous ramène rapidement sur la sphère de Riemann. Pour ces empilements de cercles, la considération des $\bmod _{\text {inf }}$ et $\bmod _{\text {sup }}$ de J.W. Cannon et d'arguments assez classiques d'estimations de modules de courbes permet de montrer simplement que les modules combinatoires et analytiques sont équivalents (Corollaire 3.8). L'intérêt de ces modules est de confronter deux familles de courbes transverses dont les modules coïncident dans le cas analytique (voir aussi le Lemme 2.14). 
On peut alors conclure par le théorème d'Ascoli pour obtenir une uniformisation globale par un homéomorphisme limite qui établit aussi la conformité de sous-suites.

Pour voir que l'homéomorphisme limite est bien quasisymétrique, et répondre au second problème, on joue sur les propriétés des recouvrements qui découlent de leur forme et de la géométrie de l'espace étudié : en particulier, on profite que la sphère de Riemann est à la fois 2-régulière et 2-loewnesque pour avoir les inégalités complémentaires à celles admises sur $X$ (cf. Prop. 3.11).

Il semblerait que les deux approches originales aient plus de points communs qu'il n'y paraît. Par exemple, J.W. Cannon considère les modules dans la surface proprement dite et, uniquement en dimension deux, alors qu'ils sont définis sur le nerf par M. Bonk et B. Kleiner, et en dimension $Q$ variable ; en pratique, ils se trouvent être équivalents dans les mêmes dimensions (Remarque 3.12 et Proposition 2.11).

De plus, J.W. Cannon et al. établissent dans [8, 10] des inégalités de type "2-régulière" qui tiennent un rôle prépondérant dans leur argumentation, et se ramènent ainsi au Corollaire 3.14. Se donner des estimations à la fois sur les $\bmod _{\text {sup }}$ et les $\bmod _{\text {inf }}$ revient à se donner à la fois des majorations et des minorations, donc des propriétés de type loewnesque et de 2-régularité. D’un autre côté, lorsque M. Bonk et B. Kleiner supposent que $X$ est 2-régulier ou se donnent les hypothèses du Corollaire 3.13, ils supposent en fait que les approximations sont séparantes, et se rapprochent ainsi du théorème de Riemann combinatoire.

Terminons par une question dont la réponse ne semble pas découler de la présente analyse :

Question 3.18. Un espace métrique $Q$-régulier $X$ homéomorphe à $\mathbb{S}^{2}, Q>2$, et muni d'une suite de recouvrements par pièces uniformément rondes, de valence bornée, dont le nerf contient une triangulation équivalente, et conforme, est-il quasisymétrique à $\widehat{\mathbb{C}}$ ?

\section{Applications à la Conjecture de CAnnon}

Ces problèmes de discrétisation que nous avons traités jusqu'ici sont motivés par la théorie géométrique des groupes et la géométrisation des variétés de dimension 3 [7]. En général, la classe de quasi-isométrie d'un groupe hyperbolique est déterminée par la topologie et la structure quasiconforme de son bord. En petite dimension, on tend à penser que la topologie seule suffit à déterminer le groupe à quasi-isométrie 
près. D'autre part, la conjecture de géométrisation W.P. Thurston implique que le groupe fondamental d'une variété compacte sans bord est hyperbolique si et seulement si la variété peut être munie d'une structure hyperbolique complète [23].

A l'intersection de ces deux problématiques, J.W. Cannon propose la conjecture suivante dans [7].

Conjecture 4.1 (de Cannon). Un groupe hyperbolique de bord homéomorphe à $\mathbb{S}^{2}$ opère géométriquement sur l'espace hyperbolique $\mathbb{H}^{3}$.

Un théorème de D. Sullivan permet de reformuler cette conjecture sous une forme plus analytique.

Conjecture 4.2. Si le bord d'un groupe hyperbolique est homéomorphe $\grave{a} \mathbb{S}^{2}$, alors il est quasisymétrique $\grave{a} \widehat{\mathbb{C}}$.

4.1. Approche de Cannon et al. Soit $G$ un groupe hyperbolique au sens de Gromov dont le bord est homéomorphe à la sphère $\mathbb{S}^{2}$. On se fixe un système fini de générateurs et on lui associe son graphe de Cayley $(X, o)$ vu comme espace métrique. On munit son bord $\partial X$ d'une métrique visuelle de paramètre $\varepsilon$. Rappelons que pour un rayon $R>0$ fixé assez grand et $x \in X$, l'ombre $\mho_{o}(x, R) \subset \partial X$ de la boule $B(x, R)$ portée par une source de lumière en $o$ ressemble à une boule de rayon $e^{-\varepsilon|x|}$. Pour chaque $n \in \mathbb{N} \backslash\{0\}$, l'ensemble $\mho_{n}=\left\{\mho_{o}(x, R),|x|=n\right\}$ détermine un quasi-empilement de $\partial X$ de valence bornée.

J.W. Cannon et E.L. Swenson ont montré qu'un groupe vérifiait la conjecture de Cannon si et seulement si la famille des recouvrements $\left\{\mho_{n}\right\}_{n}$ est conforme [11]. Ultérieurement, J.W. Cannon, W. Floyd et W. Parry ont affaibli la condition sur $\left\{\mho_{n}\right\}$ d'être conforme pour ne supposer que la variante suivante de la séparation : pour chaque point $x$ du bord et chaque voisinage $V$ de $x$, il existe un anneau $A$ qui sépare $x$ du complémentaire de $V$ et une constante $m=m(A)>0$ tels que $\bmod _{\text {inf }}\left(A, \mho_{n}\right) \geq m$ (Théorème 8.2 de $[10]$ ).

Dans leur approche, ils utilisent qu'on a a priori une structure combinatoire à l'infini qui est "la bonne" si jamais le groupe opère géométriquement sur $\mathbb{H}^{3}$. Du coup, nul besoin d'étudier les propriétés de la jauge conforme à l'infini.

4.2. Approche de Bonk et Kleiner. Dans [4, 6], M. Bonk et B. Kleiner travaillent avec des hypothèses de nature analytique. Tout d'abord, il faut savoir que le bord d'un groupe hyperbolique $G$ est toujours Ahlfors-régulier et linéairement localement connexe lorsqu'il est connexe. De plus, l'action des isométries est uniformément quasimöbius sur le bord à l'infini (les birapports de quatre points sont quasipréservés). 
M. Bonk et B. Kleiner montrent que s'il existe une métrique Ahlforsrégulière de dimension minimale $Q \geq 2$ (parmi les métriques Ahlforsrégulières de la jauge conforme) sur $\partial G$, alors la conjecture est satisfaite. Cette condition permet de montrer qu'un espace tangent (faible) $T$ de $\partial G$ admet une famille de courbes de $Q$-module non nul [17]. Or $T$ est aussi $Q$-Ahlfors-régulier et quasimöbius équivalent à un épointement de $\partial G$. Ceci implique que $\partial G$ admet aussi une famille de courbes de $Q$-module non nul (quasi-invariance du module par transformation quasimöbius [24]).

La dynamique par transformations quasimöbius permet d'exploiter cette famille de courbes pour montrer que $\partial G$ vérifie la condition de Loewner restreinte aux paires de boules de même rayon. M. Bonk et B. Kleiner montrent alors que cette condition est suffisante pour montrer que $\partial G$ est loewnesque, et ils en déduisent donc la conjecture en appliquant le Corollaire 3.17.

4.3. En guise de conclusion. Dans les deux articles [10] et [6], les auteurs partent d'une condition sur les modules de courbes : il existe une/des famille(s) de courbes de module (combinatoire) non nul : cette hypothèse - très forte - impose la présence d'une quantité importante de courbes. Ensuite, la dynamique est utilisée pour propager cette information à tous les endroits et à toutes les échelles. Cette donnée se traduit par l'existence d'une structure analytique sur $\partial G$ compatible avec ces modules et l'action de $G$. Enfin, le théorème de Sullivan produit l'action par transformations de Möbius.

On peut tirer la leçon suivante : la dynamique (cocompacte et à distorsion bornée) permet d'obtenir des estimations uniformes à toutes les échelles et dans toutes les positions à partir d'estimations plus qualitatives.

Il semblerait que l'analogie s'arrête ici.

Si l'on part du fait qu'il existe une famille de courbes de $Q$-module non nul dans un espace $Q$-régulier $(Q \geq 2)$, alors l'argument dynamique de M. Bonk et B. Kleiner évoqué plus haut permet de "balader" cette famille de courbes un peu partout. De plus, le lemme du pont et la monotonie des modules combinatoires en fonction de l'exposant $Q$ montrent que les 2-modules de condensateurs sont minorés, mais cela n'implique pas a priori la séparation combinatoire (il s'agit de l'inégalité inverse), ou alors, il faudrait montrer que ces estimations s'appliquent aux familles de courbes qui séparent les condensateurs (cf. $\bmod _{\text {inf }}$ ).

Réciproquement, si les hypothèses de séparation combinatoire sont vérifiées, on n'a aucune raison d'avoir l'existence d'une famille de courbes 
de module non nul : il se peut même qu'il n'y ait aucune courbe rectifiable sur la surface! Néanmoins, si on se donne les hypothèses de séparation combinatoire sur $\partial G$ pour un exposant $Q \geq 2$ et que l'on suppose aussi que $\partial G$ est $Q$-Ahlfors régulier, alors la Proposition B.2 montre qu'il existe une famille de courbes de module positif : on est donc ramené à [6] ! Mais les hypothèses sont très contraignantes puisque le $Q$-module combinatoire d'une famille de courbes est toujours plus petit que son 2-module, et tend même vers 0 dans les cas favorables!

En résumé, une première impression indique que l'approche de M. Bonk et $\mathrm{B}$. Kleiner est plus approfondie puisque l'on récupère des propriétés combinatoires (construction de triangulations et contrôle des modules combinatoires associés) à partir de données analytiques, alors que l'approche de J.W. Cannon et al. part directement de données combinatoires. En revanche, il est plus raisonnable de considérer des modules combinatoires, puisque l'on ne sait pas en général s'il existe des courbes rectifiables... De plus, il faut bien comprendre que la donnée initiale est celle d'un groupe hyperbolique de bord $\mathbb{S}^{2}$, donc les données sont plutôt de nature combinatoire et topologique, et les propriétés analytiques ne pourront provenir - comme c'est déjà le cas - que de considérations combinatoires...

Enfin, notons que dans les deux cas, les conditions suffisantes mises en avant (existence quantifiée de courbes) sont a priori et en général difficiles - pour ne pas dire quasi-impossibles - à vérifier... ce qui laisse le problème encore ouvert!

En effet, on sait qu'il existe des métriques $Q$-régulières sur $\partial G$, pour des exposants $Q>2$ : on sait donc comment majorer les $Q$-modules de condensateurs, mais comment montrer qu'on a une famille de $Q$ module positif? ou que sa dimension est minimale? ? De même, qu'estce qui nous permet de contrôler les 2-modules combinatoires? Comment montrer que l'on peut choisir $Q=2$ sans répondre aux questions précédentes?

\section{Annexe A. Notions de théorie GÉométrique Des Fonctions}

Des précisions sur les notions développées dans cet appendice peuvent être trouvées par exemple dans les ouvrages [1, 2, 14].

Nous nous plaçons dans un premier temps sur la sphère de Riemann. Le module est un outil fondamental de l'analyse complexe issu de la méthode longueur-aire, et ce, pour au moins deux raisons. Tout d'abord il se traduit souvent par des contraintes géométriques, et ensuite, il se comporte bien par revêtements (ramifiés) holomorphes (ou quasiconformes). 
Définition A.1 (module (analytique) de familles de courbes). Soit $\Gamma$ une famille de courbes de $\widehat{\mathbb{C}}$ et $Q>1$ un réel. On définit le $Q$-module de $\Gamma$ par

$$
\bmod _{Q} \Gamma=\inf \int_{\widehat{\mathbb{C}}} \rho^{Q}
$$

où l'infimum est pris sur toutes les fonctions boréliennes (dites admissibles) $\rho: X \rightarrow[0, \infty]$ telles que, pour toute courbe rectifiable $\gamma \in \Gamma$, $\int_{\gamma} \rho d s \geq 1$.

Lorsque $X=\widehat{\mathbb{C}}$ et $Q=2$, on obtient ainsi un invariant conforme.

Donnons quelques propriétés élémentaires du module.

(1) $\bmod _{Q}(\emptyset)=0$;

(2) si $\Gamma_{1} \subset \Gamma_{2}, \bmod _{Q} \Gamma_{1} \leq \bmod _{Q} \Gamma_{2}$;

(3) $\bmod _{Q}\left(\bigcup_{i=1}^{\infty} \Gamma_{i}\right) \leq \sum_{i=1}^{\infty} \bmod _{Q} \Gamma_{i}$;

(4) si $\Gamma_{1}$ et $\Gamma_{2}$ sont deux familles de courbes telles que toute courbe $\gamma_{1}$ dans $\Gamma_{1}$ possède une sous-courbe $\gamma_{2} \in \Gamma_{2}$, alors $\bmod _{Q} \Gamma_{1} \leq$ $\bmod _{Q} \Gamma_{2}$.

On a le critère suivant.

Proposition A.2 (critère de Beurling). Soient $\Gamma$ une famille de courbes. Une métrique $\rho$ est extrémale s'il existe une sous-famille $\Gamma_{0} \subset \Gamma$ telle que

(1) pour tout $\gamma \in \Gamma_{0}, \ell_{\rho}(\gamma)=L_{\rho}(\Gamma)$;

(2) si $h: \widehat{\mathbb{C}} \rightarrow \mathbb{R}$ vérifie $\int_{\gamma} h \geq 0$ pour toute courbe $\gamma \in \Gamma_{0}$, alors on $a \int h \rho^{Q-1} \geq 0$.

De plus, cette métrique est unique à normalisation près.

Quitte à jeter une sous-famille de courbes de module zéro, il existe une métrique extrémale, mais il n'est pas clair que la réciproque soit vraie en général.

DÉmonstration. Soit $\sigma \in \mathcal{M}_{Q}$ que l'on suppose normalisée pour que $L_{\sigma}(\Gamma)=L_{\rho}(\Gamma)$. Du coup, $\ell_{\sigma}(\gamma) \geq L_{\rho}(\Gamma)=\ell_{\rho}(\gamma)$ pour toute $\gamma \in \Gamma_{0}$ d'après (1). En posant $h=\sigma-\rho$, on obtient par (2)

$$
\int(\sigma-\rho) \rho^{Q-1} \geq 0
$$

soit

$$
\int \rho^{Q} \leq \int \sigma \rho^{Q-1} \leq\left(\int \sigma^{Q}\right)^{1 / Q} \cdot\left(\int \rho^{Q}\right)^{1-1 / Q}
$$

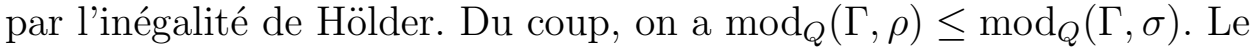
cas d'égalité se produit lorsque l'inégalité de Hölder est une égalité, 
donc si $\sigma^{Q}$ et $\left(\rho^{Q-1}\right)^{Q /(Q-1)}$ sont proportionnelles, soit si $\sigma=\rho$ presque partout d'après leurs normalisations.

Définition A.3 (module d'anneaux, de quadrilatères et de condensateurs). Si $A \subset \widehat{\mathbb{C}}$ est un anneau, on lui associe la famille $\Gamma_{t}$ des courbes qui joignent les composantes de bord, et $\Gamma_{s}$ la famille des courbes qui séparent ces composantes. On a (lemme et définition)

$$
\bmod A=\bmod _{2} \Gamma_{s}=\frac{1}{\bmod _{2} \Gamma_{t}} .
$$

Un quadrilatère $Q$ est un domaine de Jordan avec quatre points marqués sur le bord. Ces points découpent le bord en quatre "côtés" $c_{1}, c_{2}, c_{3}, c_{4}$ consécutifs. On peut définir deux modules, selon que l'on considère la famille $\Gamma_{\text {impair }}$ des courbes qui relient $c_{1}$ et $c_{3}$ ou la famille $\Gamma_{\text {pair }}$ des courbes qui relient $c_{2}$ et $c_{4}$. Similairement au cas de l'anneau, on a toujours

$$
\bmod _{2} \Gamma_{\text {impair }}=\frac{1}{\bmod _{2} \Gamma_{\text {pair }}}
$$

$U n$ condensateur est formé de deux continua $E$ et $F$ disjoints. On note $\Gamma=\Gamma(E, F)$ la famille des courbes qui relient $E$ et $F$, et on définit

$$
\bmod _{Q}(E, F)=\bmod _{Q} \Gamma .
$$

Remarque A.4. Il faut faire attention à ce que le module d'un anneau est l'inverse du module du condensateur formé des composantes du complémentaire.

Le critère de Beurling permet de montrer que dans un rectangle, le module est le rapport des longueurs des côtés, et dans le cas d'une couronne $A=\{r<|z|<R\}, \bmod A=(1 / 2 \pi) \log R / r$. En particulier, si un anneau a un très grand module, alors au moins l'une des composantes connexes de son complémentaire a un petit diamètre (cela découle p.ex. du théorème de la couronne de Teichmüller qui affirme qu'un anneau de grand module contient toujours une couronne de module comparable, voir [2, Thm 4.8]).

Les applications qui sont naturelles dans ce contexte sont les homéomorphismes quasiconformes. Ils ont la particularité de pouvoir être définis de bien des manières dans $\mathbb{C}$. Nous en citons quelques unes.

Soit $f: \mathbb{C} \rightarrow \mathbb{C}$ une application et soient $r>0$ et $z \in \mathbb{C}$; on définit

$$
\begin{aligned}
& L_{f}(z, r)=\sup \{|f(z)-f(w)|,|z-w| \leq r\} \\
& \ell_{f}(z, r)=\inf \{|f(z)-f(w)|,|z-w| \geq r\} .
\end{aligned}
$$

Si $\Gamma$ est une famille de courbes, alors $f(\Gamma)$ désigne $\{f(\gamma), \gamma \in \Gamma\}$. 
Définition A.5 (homéomorphisme quasiconforme du plan). Un homéomorphisme $f: \mathbb{C} \rightarrow \mathbb{C}$ est quasiconforme si l'une des conditions équivalentes suivantes est vérifiée.

[QC] Il existe $H<\infty$ telle que pour tout $z \in \mathbb{C}$,

$$
\limsup _{r \rightarrow 0} \frac{L_{f}(z, r)}{\ell_{f}(z, r)} \leq H .
$$

[G1] Il existe $K<\infty$ telle que pour toute famille $\Gamma$ de courbes rectifiables,

$$
(1 / K) \cdot \bmod _{2} \Gamma \leq \bmod _{2} f(\Gamma) \leq K \cdot \bmod _{2} \Gamma .
$$

[G2] Il existe $K<\infty$ telle que pour tout anneau $A \subset \mathbb{C}$,

$$
(1 / K) \cdot \bmod A \leq \bmod f(A) \leq K \cdot \bmod A .
$$

[QS] Il existe une fonction continue et croissante $\eta: \mathbb{R}_{+} \rightarrow \mathbb{R}_{+}$telle que $\eta(0)=0$, et si $z, w_{1}$ et $w_{2}$ sont trois points du plan tels que $\left|z-w_{1}\right| \leq t \cdot\left|z-w_{2}\right|$ alors

$$
\left|f(z)-f\left(w_{1}\right)\right| \leq \eta(t) \cdot\left|f(z)-f\left(w_{2}\right)\right| .
$$

[FQS] Il existe $H<\infty$ telle que pour tout $z \in \mathbb{C}$ et tout $r>0$,

$$
L_{f}(z, r) \leq H \cdot \ell_{f}(z, r) .
$$

Pour utiliser des techniques similaires dans des espaces métriques, il convient de rajouter quelques restrictions de nature géométrique. Une condition naturelle est celle d'être Ahlfors régulier. Elle est implicite dans les estimations faites sur $\widehat{\mathbb{C}}$.

Définition A.6 (Ahlfors régularité). Si $Q>0$, un espace métrique $X$ est $Q$-Ahlfors régulier s'il existe une mesure borélienne $\mu$ telle que, pour tout $R \leq \operatorname{diam} X$, et tout $x \in X$ et toute boule fermée $B(x, R)$, $\mu(B(x, R)) \asymp R^{Q}$.

Dans ce cas, on peut choisir $\mu=\mathcal{H}_{Q}$ la mesure de Hausdorff $Q$ dimensionnelle.

On remarque qu'un tel espace est toujours doublant i.e., il existe un naturel $N$ tel que tout ensemble borné peut être recouvert par au plus $N$ ensembles de diamètre moitié.

Une courbe $\gamma$ dans $X$ est une application continue d'un intervalle compact $I$ de $\mathbb{R}$ dans $X$. On peut, comme dans les espaces euclidiens, définir la longueur de $\gamma$. Si cette longueur $\ell(\gamma)$ est finie, on dira que la courbe est rectifiable. Dans ce cas, on peut paramétrer $\gamma$ par la 
longueur d'arc $\gamma_{s}$ et pour toute fonction borélienne $\rho: X \rightarrow \mathbb{R}_{+}$, on définit

$$
\int_{\gamma} \rho d s=\int_{0}^{\ell(\gamma)} \rho \circ \gamma_{s}(t) d t
$$

Cela suffit pour étendre la notion de module (analytique) de courbes aux espaces mesurés.

On conclut ces propriétés par un résultat de S. Keith (Proposition 4.2.1 [16]) qui nous sera utile dans l'appendice suivant.

Proposition A.7 (S. Keith). Soient $(X, \mu)$ un espace métrique compact de masse finie et $Q>1$. Pour toute famille de courbes $\Gamma$ et tout $\varepsilon>0$, il existe une fonction continue $g: X \rightarrow \mathbb{R}_{+}$telle que $L_{g}(\Gamma)=1$ et

$$
\int_{X} g^{Q} \leq \bmod _{Q} \Gamma+\varepsilon
$$

Le prochain lemme établit une borne supérieure sur le $Q$-module dans un espace $Q$-régulier.

Lemme A.8. Si $(X, \mu)$ est $Q$-Ahlfors régulier, $x \in X$ et $\ell<L<$ $\operatorname{diam} X$ avec $L \geq 2 \ell$, alors

$$
\bmod _{Q}(B(x, \ell), X \backslash B(x, L)) \lesssim(1 / \log L / \ell)^{Q-1}
$$

La démonstration consiste à estimer le module en utilisant la métrique

$$
\rho(y)=(1 / \operatorname{dist}(x, y)) \chi_{B(x, L) \backslash B(x, \ell)}(y) .
$$

L'estimation opposée du module n'est a priori pas automatique sous la seule condition de régularité. Son existence provient de ce que l'on appelle la condition de Loewner, qui a été introduite par J. Heinonen et P. Koskela [15] dans un contexte purement métrique. Dans ces espaces, les méthodes de discrétisation sont particulièrement pertinentes.

Définition A.9 (distance relative). Si $(E, F)$ est un condensateur dans un espace métrique, on définit leur distance relative par

$$
\Delta(E, F):=\frac{\operatorname{dist}(E, F)}{\min \{\operatorname{diam} E, \operatorname{diam} F\}} .
$$

Définition A.10 (espace loewnesque). Soit $(X, \mu)$ un espace métrique, mesuré de dimension de Hausdorff $Q$. On dit que $X$ est un espace loewnesque s'il vérifie la condition de Loewner suivante : il existe une fonction $\psi:] 0, \infty[\rightarrow] 0, \infty[$ telle que, pour tout condensateur $(E, F)$ et pour tout $t \geq \Delta(E, F)$, on ait

$$
\bmod _{Q}(E, F) \geq \psi(t) .
$$


Un espace loewnesque Ahlfors régulier vérifie de nombreuses propriétés similaires aux espaces euclidiens. Par exemple, un tel espace est toujours linéairement localement connexe et quasiconvexe i.e.,

Définition A.11 (connexité locale linéaire). Un espace métrique $X$ est linéairement localement connexe s'il existe $C>0$ telle que, pour tout $x \in X$, tout $r>0$, on ait :

(1) tout couple de points dans $B(x, r)$ appartient à un continuum contenu dans $B(x, C r)$;

(2) tout couple de points dans $X \backslash \bar{B}(x, r)$ appartient à un continuum contenu dans $X \backslash \bar{B}(x,(1 / C) r)$.

Définition A.12 (quasiconvexité). Un espace métrique $X$ est quasiconvexe s'il existe $C>0$ telle que, pour tout $x, y \in X$, il existe une courbe $\gamma$ qui relie $x$ à $y$ telle que $\ell(\gamma) \leq C|x-y|$.

Dans ce contexte, on peut aussi définir la classes des homéomorphismes quasiconformes en considérant les applications qui vérifient la condition [QC], la classe des homéomorphismes géométriquement quasiconformes qui vérifient [G1] ou [G2], la classe quasisymétrique, qui vérifient la condition [QS], ou faiblement quasisymétriques [FQS]...

Définis sur des espaces doublants et connexes, les homéomorphismes faiblement quasisymétriques sont quasisymétriques. Dans un espace de Loewner Ahlfors régulier, toutes ces définitions sont encore équivalentes (ce n'est pas le cas en général). La condition loewnesque permet notamment de passer du local au global [15].

\section{Annexe B. Discrétisation Dans les espaces MÉtriques}

On étend la notion de modules combinatoires à des espaces métriques plus généraux. Dans cet appendice, on supposera que $(X, \mu)$ est un espace métrique connexe compact localement connexe $Q$-Ahlfors régulier pour un $Q>1$ de diamètre 1. L'objectif de ce paragraphe est de montrer que l'on peut discrétiser les modules de manière raisonnable dans ce contexte (voir Proposition B.2).

On se place dans la situation suivante.

Définition B.1 (Quasi-empilement). Si X est un espace métrique, on dira qu'un recouvrement $\mathcal{S}$ est un quasi-empilement s'il existe une constante $K \geq 1$ et, pour chaque $s \in \mathcal{S}$, un point $x_{s}$ de $s$ et une taille $r_{s}>0$ tels que

(1) $B\left(x_{s}, r_{s}\right) \subset s \subset B\left(x_{s}, K \cdot r_{s}\right)$.

(2) Chaque boule interne $B\left(x_{s}, r_{s}\right)$ intersecte au plus $K$ autres boules internes $B\left(x_{s^{\prime}}, r_{s^{\prime}}\right)$. 
Un empilement de cercles n'est pas un quasi-empilement, puisqu'il ne recouvre pas l'espace. On justifie la terminologie par le fait qu'un quasi-empilement se souvient de propriétés d'un empilement : les pièces sont à peu près rondes, et (2) est une extension naturelle dans notre contexte que les boules internes soient deux à deux disjointes.

On se donne une suite de quasi-empilements $\left(\mathcal{S}_{n}\right)$ dont la maille tend vers 0 . On peut procéder ainsi : pour tout $r \in] 0,1]$ on se fixe une famille de boules fermées disjointes de $\mathcal{B}(r)=\left\{B_{i}=B\left(x_{i}, r / 5\right)\right\}_{i}$ de rayon $r / 5$ telle que $\widehat{\mathcal{B}}(r)=\left\{\widehat{B}_{i}=B\left(x_{i}, r\right)\right\}$ recouvre $X$. Puisque $X$ est Ahlfors régulier, il existe une constante $K \in \mathbb{N}$, indépendante de $r$, telle que chaque boule de $\widehat{\mathcal{B}}(r)$ intersecte au plus $K$ autres boules. En effet, si $N$ est le nombre de boules $\widehat{B}_{j}$ qui intersectent une boule $\widehat{B}$ donnée, alors on a $B_{j} \subset 3 \widehat{B}$ donc

$$
r^{Q} \gtrsim \mu(3 \widehat{B}) \geq \sum_{1 \leq j \leq N} \mu\left(B_{j}\right) \gtrsim N r^{Q}
$$

Notons enfin que chaque $\widehat{\mathcal{B}}(r)$ est un quasi-empilement et on peut prendre $\mathcal{S}_{n}=\widehat{\mathcal{B}}\left(1 / 2^{n}\right)$ et $\mathcal{B}_{n}=\mathcal{B}\left(1 / 2^{n}\right)$ avec $K=5$.

Proposition B.2. Soit $(X, \mu)$ un espace $Q$-régulier, $Q>1$, muni d'une suite de quasi-empilements dont la maille tend vers zéro. Pour $L>0$, on note $\Gamma_{L}$ les courbes de $X$ de diamètre au moins $L$. Pour $n$ assez grand, on a

$$
\bmod _{Q}\left(\Gamma_{L}, \mathcal{S}_{n}\right) \asymp \bmod _{Q} \Gamma_{L}
$$

si $\bmod _{Q} \Gamma_{L}>0$ et sinon, $\lim \bmod _{Q}\left(\Gamma_{L}, \mathcal{S}_{n}\right)=0$. De même, pour tout condensateur $(E, F)$ et pour $n$ assez grand, on a

$$
\bmod _{Q}\left(E, F, \mathcal{S}_{n}\right) \asymp \bmod _{Q}(E, F)
$$

si $\bmod _{Q}(E, F)>0$ et sinon, $\lim \bmod _{Q}\left(E, F, \mathcal{S}_{n}\right)=0$.

Une des inégalités sera un corollaire quasi-immédiat du lemme suivant, que l'on extrait de la proposition car il est plus général :

Lemme B.3. Soit $(X, \mu)$ un espace Q-régulier muni d'un K-quasiempilement $\mathcal{S}$, et soit $\Gamma$ une famille de courbes. On suppose qu'il existe une constante $\kappa>0$ telle que, pour tout $s \in \mathcal{S}$ et toute $\gamma \in \Gamma$, si $\gamma \cap s \neq \emptyset$ alors $\operatorname{diam}(\gamma \cap(2 K) \cdot B(s)) \geq \kappa \cdot \operatorname{diam} B(s)$, où $B(s)$ est la boule interne de $s$.

Alors, on a

$$
\bmod _{Q} \Gamma \lesssim \bmod _{Q}(\Gamma, \mathcal{S})
$$

Avant de démontrer ces énoncés, on rappelle un lemme qui nous sera utile. 
Lemme B.4. Soit $(X, \mu)$ un espace métrique mesuré qui vérifie la condition de doublement de volume. On considère une famille de boules $B \in \mathcal{B}$ et on associe à chacune un poids $a_{B}>0$. Pour tout $p>1$, pour tout $\lambda \in] 0,1[$, il existe une constante $C=C(p, \lambda)>0$ indépendante de $\mathcal{B}$ et des poids, telle que

$$
\int\left(\sum a_{B} \chi_{B}\right)^{p} d \mu \leq C \int\left(\sum a_{B} \chi_{\lambda B}\right)^{p} d \mu
$$

La démonstration de ce lemme utilise les fonctions maximales de Hardy-Littlewood et consiste à estimer la norme de cette fonction en tant qu'élément du dual de $L^{q}$, où $(1 / p)+(1 / q)=1$ (voir [3] dans le cadre euclidien).

Démonstration Du Lemme B.3. Si $\rho \in \mathcal{M}_{Q}(X, \mathcal{S})$, on définit la soupe $\widehat{\rho}$ du poids $\rho$ par

$$
\widehat{\rho}=\sum_{s \in \mathcal{S}} \frac{\rho(s)}{\operatorname{diam} B(s)} \chi_{2 K B(s)},
$$

où $\chi_{2 K B(s)}$ désigne la fonction caractéristique de $2 K B(s)$. Du coup, si $\gamma \in \Gamma$, alors

$$
\begin{aligned}
\ell_{\widehat{\rho}}(\gamma) & \geq \sum_{s \in \mathcal{S}(\gamma)} \int_{\gamma \cap 2 K B(s)} \frac{\rho(s)}{\operatorname{diam} s} \\
& \geq \kappa \sum_{s \in \mathcal{S}(\gamma)} \rho(s) \\
& \geq \kappa L_{\rho}(\Gamma, \mathcal{S}) .
\end{aligned}
$$

D'autre part,

$$
\begin{aligned}
\mathrm{V}_{Q}(\widehat{\rho}) & =\int_{X}\left(\sum_{s \in \mathcal{S}} \frac{\rho(s)}{\operatorname{diam} s} \chi_{2 K B(s)}\right)^{Q} \\
& \lesssim \int_{X}\left(\sum_{s \in \mathcal{S}} \frac{\rho(s)}{\operatorname{diam} s} \chi_{B(s)}\right)^{Q}
\end{aligned}
$$


par le Lemme B.4. Or, pour tout $x \in X$, on a

$$
\begin{aligned}
\left(\sum_{s \in \mathcal{S}} \frac{\rho(s)}{\operatorname{diam} s} \chi_{B(s)}(x)\right)^{Q} & \leq\left((K+1) \cdot \max \left\{\frac{\rho(s)}{\operatorname{diam} s} \chi_{B(s)}(x)\right\}\right)^{Q} \\
& \leq(K+1)^{Q} \sum_{s \in \mathcal{S}}\left(\frac{\rho(s)}{\operatorname{diam} s} \chi_{B(s)}(x)\right)^{Q} .
\end{aligned}
$$

Par conséquent, on a

$$
\begin{aligned}
\mathrm{V}_{Q}(\widehat{\rho}) & \lesssim \sum_{s \in \mathcal{S}} \int_{B(s)}\left(\frac{\rho(s)}{\operatorname{diam} s}\right)^{Q} \\
& \lesssim \sum_{s \in \mathcal{S}} \rho(s)^{Q}
\end{aligned}
$$

car $\mu(B(s)) \asymp \operatorname{diam} B(s)^{Q}$. Donc

$$
V_{Q}(\widehat{\rho}) \lesssim V_{Q}(\rho, \mathcal{S})
$$

et on obtient ainsi

$$
\bmod _{Q} \Gamma \lesssim \bmod _{Q}(\Gamma, \mathcal{S})
$$

Pour l'autre inégalité, nous allons adapter l'argument de la Proposition 3.2.1 de [17]. Dans ce but, on définit une $\mathcal{S}_{n}$-approximation d'une courbe $\gamma:[0,1] \rightarrow X$ comme une suite finie de pièces $\left\{s_{1}, \ldots, s_{k}\right\}$ de $\mathcal{S}_{n}$ deux à deux distinctes, telle qu'il existe $0=t_{0}<t_{1}<\ldots<$ $t_{j}<\ldots<t_{k}=1$ qui vérifient $\left\{\gamma\left(t_{j}\right), \gamma\left(t_{j+1}\right)\right\} \subset s_{j+1}$. On note $\ell_{n}(\gamma)=\sum_{0 \leq j<k} d\left(\gamma\left(t_{j}\right), \gamma\left(t_{j+1}\right)\right)$ et on définit l'application $a_{n}(\gamma)$ : $\left[0, \ell_{n}(\gamma)\right] \rightarrow X$, constante par morceaux, par

$$
a_{n}(\gamma)\left(\left[\sum_{0 \leq j<m} d\left(\gamma\left(t_{j}\right), \gamma\left(t_{j+1}\right)\right), \sum_{0 \leq j \leq m} d\left(\gamma\left(t_{j}\right), \gamma\left(t_{j+1}\right)\right)[)=\gamma\left(t_{m}\right)\right.\right.
$$

pour $0 \leq m \leq k-1$ et $a_{n}(\gamma)\left(\ell_{n}(\gamma)\right)=\gamma(1)$.

Notons qu'en général il existe plusieurs $\mathcal{S}_{n}$-approximations d'une même courbe. Cependant, on a toujours $\ell_{n}(\gamma) \leq \sum_{\mathcal{S}_{n}(\gamma)}$ diam $s$.

Démonstration de la Proposition B.2. On se fixe un condensateur $(E, F)$ et un diamètre $L>0$, et on note $\Gamma=\Gamma(E, F)$ ou $\Gamma=\Gamma_{L}$.

On montre dans un premier temps que $\bmod _{Q} \Gamma \lesssim \bmod _{Q}\left(\Gamma, \mathcal{S}_{n}\right)$ pour $n$ assez grand, où les constantes implicites ne dépendent ni de $n$, ni du condensateur. Par hypothèses, il existe une constante $K \geq 1$ et pour chaque $s \in \cup \mathcal{S}_{n}$ une boule $B(s)$ telles que $B(s) \subset s \subset K \cdot B(s)$. Comme 
la maille de $\mathcal{S}_{n}$ tend vers 0 , il existe $\kappa>0$ tel que, pour tout $n$ assez grand, pour toute $\gamma \in \Gamma$ et toute $s \in \mathcal{S}(\gamma)$,

$$
\operatorname{diam}(\gamma \cap(2 K) \cdot B(s)) \geq \kappa \cdot \operatorname{diam} B(s) .
$$

Pour ces $n$-ci, on obtient $\bmod _{Q} \Gamma \lesssim \bmod _{Q}\left(\Gamma, \mathcal{S}_{n}\right)$ par le Lemme B.3.

Quant à la réciproque, on se fixe $\varepsilon>0$. D'après la Proposition A.7, il existe une fonction continue $g: X \rightarrow \mathbb{R}_{+}$telle que $L_{g}(\Gamma)=1$ et

$$
\int_{X} g^{Q} d \mu \leq \bmod _{Q} \Gamma+\varepsilon
$$

On peut supposer $g(x) \geq \delta>0$ puisque $X$ est compact et $\mu(X)<\infty$. On définit, pour $x \in X$,

$$
g_{n}(x)=\inf \left\{g(y), y \in s, x \in s, s \in \mathcal{S}_{n}\right\}
$$

Puisque $g$ est continue et la maille de $\mathcal{S}_{n}$ tend vers 0 , la suite $\left(g_{n}\right)$ tend uniformément vers $g$.

Notons $\rho_{n}(s):=(3 / 2) \inf _{s} g \cdot \operatorname{diam} s$, et montrons que $L_{\rho_{n}}\left(\Gamma, \mathcal{S}_{n}\right) \geq 1$ pour $n$ assez grand. Si ce n'est pas le cas, alors il existe une sous-suite $\left(n_{p}\right)$ ainsi que des courbes $\left(\gamma_{p}\right)$ de $\Gamma$ telles que $\ell_{\rho_{n_{p}}}\left(\gamma_{p}, \mathcal{S}_{n_{p}}\right)<1$. On se fixe des approximations $a_{n_{p}}\left(\gamma_{p}\right)$. Ceci implique que d'une part

$$
\sum_{\mathcal{S}_{n_{p}}\left(\gamma_{p}\right)} \operatorname{diam} s \cdot \inf _{s} g<1-(\delta / 2) \sum_{\mathcal{S}_{n_{p}}\left(\gamma_{p}\right)} \operatorname{diam} s
$$

et d'autre part $\ell_{n_{p}}\left(\gamma_{p}\right)<1 / \delta$.

Notons $\ell=\sup _{p} \ell_{n_{p}}\left(\gamma_{p}\right)$ et étendons $a_{n_{p}}\left(\gamma_{p}\right)$ à $[0, \ell]$ en posant $a_{n_{p}}\left(\gamma_{p}\right)\left(\left[\ell_{n_{p}}\left(\gamma_{p}\right), \ell\right]\right)=$ $a_{n_{p}}\left(\gamma_{p}\right)\left(\ell_{n_{p}}\left(\gamma_{p}\right)\right)$.

On obtient ainsi une suite relativement compacte pour la convergence uniforme dont toute limite est une application 1-lipschitzienne (argument similaire au théorème d'Arzéla-Ascoli). Soit $\gamma$ l'une d'elles : il s'agit, par construction, d'une courbe $\gamma \in \Gamma$ : dans le premier cas, elle relie $E$ et $F$; dans le second, son diamètre est bien minoré par $L$. 
On obtient, par le lemme de Fatou,

$$
\begin{aligned}
\int_{\gamma} g d s & \leq \liminf \int_{0}^{L} g_{n_{p}} \circ a_{n_{p}}\left(\gamma_{p}\right) \\
& \leq \liminf \sum_{\mathcal{S}_{n_{p}}\left(\gamma_{p}\right)} \operatorname{diam} s \inf _{s} g \\
& \leq 1-(\delta / 2) \limsup \sum_{\mathcal{S}_{n_{p}}\left(\gamma_{p}\right)} \operatorname{diam} s \\
& \leq 1-(\delta / 2) \ell(\gamma) .
\end{aligned}
$$

Ceci contredit $L_{g}(\Gamma)=1$.

Du coup, pour $n$ assez grand, on a

$$
\begin{aligned}
\bmod _{Q}\left(\Gamma, \mathcal{S}_{n}\right) & \lesssim \sum\left(\inf _{s} g\right)^{Q} \cdot(\operatorname{diam} s)^{Q} \\
& \lesssim \sum\left(\inf _{s} g\right)^{Q} \cdot \mu(B(s)) \\
& \leq \sum \int_{B(s)} g^{Q} d \mu \\
& \lesssim \int_{X} g^{Q} d \mu \\
& \leq \bmod _{Q} \Gamma+\varepsilon
\end{aligned}
$$

Par conséquent, si $\bmod _{Q} \Gamma=0$, on obtient $\bmod _{Q}\left(\Gamma, \mathcal{S}_{n}\right) \rightarrow 0$ avec $n$, et si $\bmod _{Q} \Gamma>0$, on obtient $\bmod _{Q}\left(\Gamma, \mathcal{S}_{n}\right) \lesssim \bmod _{Q} \Gamma$ en prenant e.g. $\varepsilon=\bmod _{Q} \Gamma$.

Cette proposition permet de donner une nouvelle démonstration qu'une application quasimöbius entre $Q$-espaces réguliers quasipréserve les $Q$ modules de condensateurs quantitativement [24]. Rappelons, suivant J. Väisälä [25], qu'un homéomorphisme $\phi: X \rightarrow Y$ entre espaces métriques est quasimöbius s'il existe un homéomorphisme $\eta: \mathbb{R}_{+} \rightarrow \mathbb{R}_{+}$ tel que, quelque soit le quadruplet de points distincts $x_{1}, x_{2}, x_{3}, x_{4} \in X$, on a

$$
\frac{\left|\phi\left(x_{1}\right)-\phi\left(x_{2}\right)\right|}{\left|\phi\left(x_{1}\right)-\phi\left(x_{3}\right)\right|} \frac{\left|\phi\left(x_{3}\right)-\phi\left(x_{4}\right)\right|}{\left|\phi\left(x_{3}\right)-\phi\left(x_{4}\right)\right|} \leq \eta\left(\frac{\left|x_{1}-x_{2}\right|}{\left|x_{1}-x_{3}\right|} \frac{\left|x_{3}-x_{4}\right|}{\left|x_{3}-x_{4}\right|}\right) .
$$

Théorème B.5 (J. Tyson). Soient $X, Y$ deux espaces compacts $Q$ réguliers et $\phi: X \rightarrow Y$ un homéomorphisme quasimöbius. Alors, pour 
tout condensateur $(E, F)$, on a

$$
\bmod _{Q}(E, F) \asymp \bmod _{Q}(\phi E, \phi F),
$$

où les constantes implicites ne dépendent que des constantes des propriétés évoquées sur $X$ et $Y$.

DÉmonstration. On se fixe une suite de recouvrements $\left(\mathcal{S}_{n}\right)$ comme pour la Proposition B.2. Une application quasimöbius est localement quasisymétrique (quantitativement) donc l'image des $\mathcal{S}_{n}$ reste une suite de recouvrements de valence bornée par pièces uniformément rondes dont la maille tend vers 0, donc, en utilisant deux fois la Proposition B.2 pour $n$ assez grand, on obtient

$\bmod _{Q}(E, F) \asymp \bmod _{Q}\left(E, F, \mathcal{S}_{n}\right)=\bmod _{Q}\left(\phi E, \phi F, \phi \mathcal{S}_{n}\right) \asymp \bmod _{Q}(\phi E, \phi F)$ ce qui établit le théorème.

\section{Annexe C. Convergence et topologie de Hausdorff}

Cet appendice précise les notions de convergence utilisées notamment dans la démonstration du Théorème 3.4.

Soit $(Z, d)$ un espace métrique. Si $X, Y \subset Z$, on note

$$
\partial(X, Y)=\sup _{x \in X} d(x, Y) .
$$

On a $\partial(X, Y)=0$ si et seulement si $\bar{X} \subset \bar{Y}$.

On définit par

$$
d_{H}(X, Y)=\max \{\partial(X, Y), \partial(Y, X)\}
$$

la distance de Hausdorff entre $X$ et $Y$. On dira qu'une suite $\left(X_{n}\right)_{n}$ de parties de $Z$ tend vers $X$ si $d_{H}\left(X_{n}, X\right) \rightarrow 0$.

En notant $V_{\varepsilon}(X)=\{x \in Z, d(x, X)<\varepsilon\}$, cela signifie que, pour tout $\varepsilon>0$, il existe $n_{0}$ tel que si $n \geq n_{0}$ alors $X_{n} \subset V_{\varepsilon}(X)$ et $X \subset V_{\varepsilon}\left(X_{n}\right)$.

Rappelons que si $X$ un espace métrique propre, alors l'ensemble des compacts non vides de $X$ muni de la distance de Hausdorff est un espace métrique complet.

Dans la démonstration du Théorème 3.4, les ensembles $\mathcal{P}_{n}$ tendent dans la topologie de Hausdorff vers $X$.

Dans un premier temps, on réinterprète la convergence au sens de Hausdorff à l'aide de la convergence uniforme de fonctions. Ensuite, on établit un théorème de convergence de fonctions définies sur des sous-espaces convergents. On se place dans un contexte très proche de ce dont on a besoin. Cette approche est inspirée par les travaux de M. Gromov sur la métrique de « Hausdorff-Gromov» dans [13], et nos 
résultats s'étendent à ce contexte plus général sans problème particulier.

Lemme C.1. Si $Z$ est un espace métrique compact, alors, pour tout $\varepsilon>0$, il existe un nombre $N(\varepsilon)$ tel que tout compact $K$ de $Z$ peut être recouvert par $N(\varepsilon)$ boules de rayon $\varepsilon$ centrées sur $K$.

DÉmonstration. Si $\varepsilon>0$ est fixé, on considère un recouvrement fini de $Z$ par des boules de rayon $\varepsilon / 2$. On note $N(\varepsilon)$ le nombre de ces boules. Pour chacune de ces boules $B$, pour chaque $x \in B$, on a $B \subset B(x, \varepsilon)$. Donc, si $K$ est compact, alors il est recouvert par au plus $N(\varepsilon)$ boules de rayon $\varepsilon$.

Soit $Z$ un espace métrique compact. On considère la suite $\left(N_{k}\right)_{k \geq 1}$ des entiers obtenus par le lemme précédent en prenant $\varepsilon=1 / 2^{k}$.

On note

$$
A_{k}=\left\{\left(n_{j}\right)_{1 \leq j \leq k} \in \prod_{1 \leq j \leq k}\left[1, N_{j}\right]\right\} \quad \text { et } p_{k}: A_{k+1} \rightarrow A_{k}
$$

l'application $\left(n_{j}\right)_{1 \leq j \leq k+1} \mapsto\left(n_{j}\right)_{1 \leq j \leq k}$. On définit

$$
A=\lim _{\longleftarrow}\left(A_{k}, p_{k}\right)=\left\{\left(a_{k}\right) \in \prod_{k \geq 1} A_{k}, p_{k}\left(a_{k+1}\right)=a_{k}\right\} .
$$

Cet espace est compact, et on peut définir une distance ultra-métrique $d_{A}$ comme suit.

$$
d_{A}\left(\left(a_{k}\right),\left(b_{k}\right)\right)=\frac{1}{2^{\min \left\{j, a_{j} \neq b_{j}\right\}}} .
$$

Lemme C.2. Si $K$ est un compact de $Z$, alors il existe une application $I: A \rightarrow K$ surjective et 2-Lipschitz.

DÉmonstration. Soit $\mathcal{B}^{1}=\left\{B_{j}^{1}\right\}_{1 \leq j \leq N_{1}}$ un recouvrement par $N_{1}$ boules de rayon $1 / 2$. On définit $I_{1}(n)=x_{n}$, le centre de $B_{n}^{1}$.

On suppose que, jusqu'au rang $n \geq 1$, on a construit des applications $I_{k}: A_{k} \rightarrow K$ telles que

(a) $I_{k}\left(A_{k}\right)$ est une $\left(1 / 2^{k}\right)$-approximation de $K$ (chaque point de $K$ est à distance au plus $1 / 2^{k}$ de $I_{k}\left(A_{k}\right)$;

(b) Pour chaque $k, I_{k+1}(a) \in B\left(I_{k} p_{k}(a), 1 / 2^{k}\right)$, pour tout $a \in A_{k+1}$.

Pour chaque $a \in A_{n}, B_{a}=B\left(I_{n}(a), 1 / 2^{n}\right)$ est recouvert par $N_{n+1}$ boules

$$
\mathcal{B}_{a}=\left\{B\left(x_{j}^{a}, 1 / 2^{n+1}\right)\right\}_{1 \leq j \leq N_{n+1}} .
$$

On définit $I_{n+1}(a, j)=x_{j}^{a}$. Notons que $x_{j}^{a} \in B_{a}$. Par définition, $I_{n+1}\left(\{a\} \times\left[1, N_{n+1}\right]\right)$ est une $1 / 2^{n+1}$-approximation de $B_{a}$. Puisque ces 
boules recouvrent $K$, on obtient (a) pour $k=n+1$. De même, (b) est vérifié par construction puisque $p_{n}(a, j)=a$.

D'après (b), si $a=\left(a_{k}\right) \in A$, alors $\left(I_{k}\left(a_{k}\right)\right)_{k}$ est une suite de Cauchy. On définit alors

$$
I(a)=\lim I_{k}\left(a_{k}\right) .
$$

On note que $I(a) \in B\left(I_{k}\left(a_{k}\right), 1 / 2^{k}\right)$ pour chaque $k$.

D'après (a) et (b), pour tout $x \in K$, on peut trouver une suite de boules dans chaque recouvrement qui contiennent $x$. Autrement dit, $I$ est surjective.

Soient $a=\left(a_{n}\right)$ et $b=\left(b_{n}\right)$ des points de $A$. On suppose que $d_{A}(a, b)=1 / 2^{k}$. Du coup, $a_{k}=b_{k}$, et $I(a), I(b) \in B\left(I_{k}\left(a_{k}\right), 1 / 2^{k}\right)$, donc $|I(a)-I(b)| \leq 1 / 2^{k-1} \leq 2 d_{A}(a, b)$.

Corollaire C.3. Si $\left(X_{n}\right)$ est une suite de compacts de $Z$ qui tend vers un compact $X$, alors, quitte à extraire une sous-suite, la suite $\left(I_{n}\right)$ définie par le Lemme C.2 tend vers une application $I: A \rightarrow Z$ telle que $I(A)=X$.

DÉmonstration. Le lemme précédent nous construit un espace métrique $A$ et une suite d'applications $I^{n}: A \rightarrow Z$ 2-lipschitziennes telles que $I^{n}(A)=X_{n}$. Cette suite est donc équicontinue et le théorème d'Ascoli nous extrait une sous-suite $\left(n_{k}\right)$ de sorte que $\left(I^{n_{k}}\right)_{k}$ est convergente vers une application 2-lipschitzienne $I: A \rightarrow Z$. Il reste à vérifier que $X=I(A)$. Or, on a

$$
d_{H}\left(X_{n_{k}}, I(A)\right) \leq \sup _{a \in A}\left|I^{n_{k}}(a)-I(a)\right|
$$

donc $I(A)$ est la limite de Hausdorff de $\left(X_{n_{k}}\right)$, ce qui établit le corollaire, puisqu'il y a unicité de la limite (parmi les ensembles fermés).

Convergence des fonctions. On étudie maintenant les limites de fonctions lorsque les espaces sont convergents.

On utilise la notion de module de continuité pour traduire la notion de continuité uniforme.

Définition C.4 (module de continuité). Si $f: X \rightarrow Y$ est continue, un module de continuité est une fonction $\omega: \mathbb{R}_{+} \rightarrow \mathbb{R}_{+}$telle que $\omega(t) \rightarrow 0$ avec $t$ et, pour tous $x, y \in X$,

$$
|f(x)-f(y)| \leq \omega(|x-y|) .
$$


On remarque aussi que si une famille de fonctions est uniformément équicontinue, il existe un module de continuité qui ne dépend pas de la fonction de la famille.

Definition. - Soient $Z$ et $Y$ deux espaces métriques compacts, $\left(X_{n}\right)$ une suite de compacts de $Z$ qui converge vers un compact $X$. Soit $\left(f_{n}: X_{n} \rightarrow Y\right)$ une suite d'applications continues. On dit que $\left(f_{n}\right)$ converge vers $f: X \rightarrow Y$ si, pour tout $\varepsilon>0$ et pour tout $z \in X$, il existe $\alpha>0$, tel que, pour tout $n$ assez grand, si $z_{n} \in X_{n}$ vérifie $d\left(z_{n}, z\right)<\alpha$ alors $d\left(f_{n}\left(z_{n}\right), f(z)\right)<\varepsilon$.

On obtient ainsi

Théorème C.5. Soient $Z$ et $Y$ deux espaces métriques compacts, $\left(X_{n}\right)$ une suite de compacts de $Z$ qui converge vers un compact $X$. Soit $\left(f_{n}\right.$ : $\left.X_{n} \rightarrow Y\right)$ une suite d'applications continues qui admet un module de continuité uniforme.

Il existe une sous-suite $\left(f_{n_{k}}\right)$ qui converge vers une application continue $f: X \rightarrow Y$, munie du même module de continuité.

En particulier, si la suite $\left(f_{n}\right)$ est isométrique, L-Lipschitz ou $\eta$ quasisymétrique, il en est de même de la limite.

DÉmonstration. Soit $\omega$ le module de continuité uniforme de $\left(f_{n}\right)$. On note $A$ le compact obtenu par le Lemme C.2 pour $X_{n}$ et on suppose que $I^{n}$ tend vers $I$ uniformément.

On obtient ainsi une suite d'applications $g_{n}=f_{n} \circ I^{n}: A \rightarrow Y$ uniformément équicontinue.

Le théorème d'Ascoli nous permet d'extraire une sous-suite convergente vers une application $g: A \rightarrow Y$.

Soient $z, w \in X$. Il existe $a, b \in A$ tel que $I(a)=z$ et $I(b)=w$. Du coup,

$|g(a)-g(b)|=\left|f_{n}\left(I^{n}(a)\right)-f_{n}\left(I^{n}(b)\right)\right| \pm\left(\left|g_{n}(a)-g(a)\right|+\left|g_{n}(b)-g(b)\right|\right)$

donc

$$
|g(a)-g(b)|=\lim \left|f_{n}\left(I_{n}(a)\right)-f_{n}\left(I_{n}(b)\right)\right| .
$$

Du coup, $g(a)=g(b)$ si $I(a)=I(b)$. Autrement dit, il existe $f$ : $X \rightarrow Y$ telle que $g=f \circ I$. Cette application $f$ a le même module de continuité :

$|f(z)-f(w)|=\lim \left|f_{n}\left(I^{n}(a)\right)-f_{n}\left(I^{n}(b)\right)\right| \leq \lim \omega\left(\left|I^{n}(a)-I^{n}(b)\right|\right)=\omega(|z-w|)$.

Le reste suit, et est laissé au lecteur. 


\section{RÉFÉRENCES}

[1] L.V. Ahlfors, Lectures on quasiconformal mappings, Van Nostrand, 1966.

[2] L.V.Ahlfors, Conformal invariants : topics in geometric function theory, McGraw-Hill Series in Higher Mathematics. McGraw-Hill Book Co., New York-Düsseldorf-Johannesburg, 1973.

[3] B. Bojarski, Remarks on Sobolev imbedding inequalities, Complex analysis, Joensuu 1987, 52-68, Lecture Notes in Math., 1351, Springer, Berlin-New York, 1988.

[4] M. Bonk \& B. Kleiner, Rigidity for quasimöbius group actions, J. Differential Geom. 61 (2002), no. 1, 81-106.

[5] M. Bonk \& B. Kleiner, Quasisymmetric parametrizations of two-dimensional metric spheres, Invent. Math. 150 (2002), no. 1, 127-183.

[6] M. Bonk \& B. Kleiner, Conformal dimension and Gromov hyperbolic groups with 2-sphere boundary, Geom. Topol. 9 (2005), Paper no. 7, 219-246.

[7] J.W. Cannon, The theory of negatively curved spaces and groups, Ergodic theory, symbolic dynamics, and hyperbolic spaces (Trieste, 1989), 315-369, Oxford Sci. Publ., Oxford Univ. Press, New York, 1991.

[8] J.W.Cannon, The combinatorial Riemann mapping theorem, Acta Math. 173 (1994), no. 2, 155-234.

[9] J.W. Cannon, W.J. Floyd \& W.R. Parry, Squaring rectangles : the finite Riemann mapping theorem, The mathematical legacy of Wilhelm Magnus : groups, geometry and special functions (Brooklyn, NY, 1992), 133-212, Contemp. Math., 169, Amer. Math. Soc., Providence, RI, 1994.

[10] J.W. Cannon, W.J. Floyd \& W.R. Parry, Sufficiently rich families of planar rings, Ann. Acad. Sci. Fenn. Math. 24 (1999), no. 2, 265-304.

[11] J.W. Cannon \& E.L.Swenson, Recognizing constant curvature discrete groups in dimension 3, Trans. Amer. Math. Soc. 350 (1998), no. 2, 809849 .

[12] Y. Colin de Verdière, Un principe variationnel pour les empilements de cercles, Invent. Math. 104 (1991), no. 3, 655-669.

[13] M. Gromov, Groups of polynomial growth and expanding maps, Inst. Hautes Études Sci. Publ. Math. 53 (1981), 53-73.

[14] J. Heinonen, Lectures on analysis on metric spaces, Universitext. SpringerVerlag, New York, 2001.

[15] J.Heinonen \& P. Koskela, Quasiconformal maps in metric spaces with controlled geometry, Acta Math. 181 (1998), no. 1, 1-61.

[16] S. Keith, Modulus and the Poincaré inequality on metric measure spaces, Math. Z. 245 (2003), no. 2, 255-292.

[17] S. Keith \& T. Laakso, Conformal Assouad dimension and modulus, Geometric and Functional Analysis, 14 (2004), no. 6, 1278-1321.

[18] P. Koebe, Kontaktprobleme der konformen Abbildung, Ber. Sächs. Akad. Wiss. Leipzig, Math.-phys 88 (1936), 141-164. 
[19] J. Nagata, Modern dimension theory Bibliotheca Mathematica, Vol. VI. Edited with the cooperation of the "Mathematisch Centrum" and the "Wiskundig Genootschap" at Amsterdam Interscience Publishers John Wiley \& Sons, Inc., New York 1965.

[20] P. Pansu, Dimension conforme et sphère à l'infini des variétés à courbure négative. Ann. Acad. Sci. Fenn. Ser. A I Math. 14 (1989), no. 2, 177-212.

[21] B. Rodin \& D. Sullivan, The convergence of circle packings to the Riemann mapping, J. Differential Geom. 26 (1987), no. 2, 349-360.

[22] O. Schramm, Square tilings with prescribed combinatorics, Israel J. Math. 84 (1993), no. 1-2, 97-118.

[23] W.P. Thurston, Three-dimensional manifolds, Kleinian groups and hyperbolic geometry, Bull. Amer. Math. Soc. (N.S.) 6 (1982), no. 3, 357-381.

[24] J.T.Tyson, Metric and geometric quasiconformality in Ahlfors regular Loewner spaces, Conform. Geom. Dyn. 5 (2001), 21-73.

[25] J. Väisälä, Quasi-Möbius maps, J. Analyse Math. 44 (1984/85), 218-234.

LATP/CMi, Université de Provence, 39, Rue Frédéric Joliot-Curie, 13453 MARseille CEDEX 13, FranCE

E-mail address: phaissin@cmi.univ-mrs.fr 